\title{
Magnetic Resonance RF pulse design by optimal control with physical constraints
}

\author{
Armin Rund ${ }^{*}, \dagger$ \\ Christoph Stefan Aigner \\ Karl Kunisch ${ }^{*} \S$ \\ Rudolf Stollberger ${ }^{\text {t十 }}$
}

\begin{abstract}
Optimal control approaches have proved useful in designing RF pulses for large tip-angle applications. A typical challenge for optimal control design is the inclusion of constraints resulting from physiological or technical limitations, that assure the realizability of the optimized pulses. In this work we show how to treat such inequality constraints, in particular, amplitude constraints on the B1 field, the slice-selective gradient and its slew rate, as well as constraints on the slice profile accuracy. For the latter a pointwise profile error and additional phase constraints are prescribed. Here, a penalization method is introduced that corresponds to a higher-order tracking instead of the common quadratic tracking. The order is driven to infinity in the course of the optimization. We jointly optimize for the RF and slice-selective gradient waveform. The amplitude constraints on these control variables are treated efficiently by semismooth Newton or quasi-Newton methods.

The method is flexible, adapting to many optimization goals. As an application we reduce the power of refocusing pulses, which is important for spin echo based applications with a short echo spacing. Here, the optimization method is tested in numerical experiments for reducing the pulse power of simultaneous multislice refocusing pulses. The results are validated by phantom and in-vivo experiments.
\end{abstract}

Keywords: RF pulse design, slice-selective, optimal control, physical constraints, inequality constraints

\section{Introduction}

Magnetic resonance imaging at high field strength is restricted by the specific absorption rate (SAR) and technical limitations of the hardware. For single transmit MR imaging the SAR is proportional to the overall or global pulse power. SAR limitations apply mainly for sequences with larger flip angles (e.g. turbo or multi spin echo) or short TR (e.g. True FISP, SSFP).

Various methods exist to design slice-selective RF pulses [6]. For small flip angles the Bloch equation can be approximated by the inverse Fourier transform, while large flip angles require the full Bloch equation. Neglecting the relaxation terms and assuming pointwise constancy, the

*Institute for Mathematics and Scientific Computing, University of Graz, Heinrichstrasse 36, 8010 Graz, Austria (armin.rund@uni-graz.at)

†BioTechMed Graz, 8o10 Graz, Austria

¥Institute of Medical Engineering, Graz University of Technology, Stremayrgasse 16/III, 8010 Graz, Austria

§Johann Radon Institute for Computational and Applied Mathematics (RICAM), Austrian Academy of Sciences, Altenbergstraße 69, 4040 Linz, Austria 
Bloch equation can be expressed in the spin domain [19] and inverted by the Shinnar Le-Roux transform for large tip angle RF pulse design [25].

In the context of Simultaneous Multislice (SMS) imaging [3] the global RF power typically increases linearly with the multiband (MB) factor, while the increase in the maximum $B_{1}$ peak amplitude can be reduced by phase scrambling [34], time shifting [2] or root flipping [28]. Using sampling and replication properties from the Fourier transform, the PINS method [24] allows for a refocusing of many periodic slices without a power increase at the cost of a reduced bandwith and long pulse durations, which makes PINS or its enhancement MultiPINS [10] the gold standard for large MB factors. A different approach to reduce the power of a given RF pulse with a distinct slice profile can be accomplished by applying the VERSE principle $[8,15,20]$. Alternatively, the pulse can be improved iteratively by optimal control approaches $[1,12,21,33]$. Here, one typically minimizes a quadratic objective subject to the Bloch equations.

The aim of this work is to introduce new models and optimal control methods for minimizing the global RF power while restricting the maximal slice profile error. To effectively reduce the pulse power, both the RF pulse and the slice-selective gradient (Gs) are controlled jointly. For ensuring the practical applicability of the optimized pulses, technical constraints on the MR hardware are included into the optimization. Among these are amplitude constraints on Gs and its slew rate, as well as amplitude constraints on the RF pulse. In contrast to existing optimal control approaches, the proper excitation/refocusing pattern is modeled in a detailed way using an error band around a desired pattern. Accordingly we cast the design objectives as inequality constraints rather than as quadratic tracking type functionals. New methods for solving the resulting inequality-constrained optimal control problems for RF pulse design are introduced. Since a reduced pulse power is highly important especially in SMS acquisition [3], test examples from this field are chosen for numerical experiments, as well as phantom and in-vivo measurements.

In our preceding work [1] we set up efficient second-order optimization methods for optimal control of the Bloch equations with relaxation. Here we show how to extend second-order methods to different types of inequality constraints. Semismooth Newton and quasi-Newton methods are introduced and combined with new penalization techniques for assuring the profile accuracy. Furthermore, we extend the optimization framework to the common spin-domain description $[19,25]$ at the cost of neglecting the relaxation.

\section{Theory}

The nuclear magnetization vector $\mathbf{M}$ is described by the Bloch equation (without relaxation) in the on-resonance case $\dot{\mathbf{M}}(t, z)=\gamma \mathbf{B}(t, z) \times \mathbf{M}(t, z)$. The external magnetic field $\mathbf{B}(t, z)=$ $\left(\operatorname{Re}\left(B_{1}(t)\right), \operatorname{Im}\left(B_{1}(t)\right), g(t) z\right)$ depends on the complex-valued RF pulse $B_{1}(t)$ and the amplitude $g(t)$ of Gs, as well as the spatial position $z$ and time $t \in(0, T)$. The aim of the optimization will be to control $B_{1}(t)$ and $g(t)$ jointly in order to approximately reach a space-dependent desired magnetization pattern at the terminal time $T$ with a minimum pulse power. The optimization model will be defined in the spin domain.

\subsection{Spin domain Bloch equation}

Assuming the external magnetic field to be piecewise constant in time, this Bloch equation can be solved in the spin domain $[19,25]$ as a sequence of rotations, where the magnetization vector 
$\mathbf{M}$ can be described by the complex-valued Cayley-Klein parameters $\left(a_{m}\right),\left(b_{m}\right), m=1, \ldots, N_{t}$ with evolution

$$
\begin{aligned}
& a_{m}=\alpha_{m} a_{m-1}-\beta_{m}^{*} b_{m-1}, \\
& b_{m}=\beta_{m} a_{m-1}+\alpha_{m}^{*} b_{m-1},
\end{aligned}
$$

and with initial conditions $a_{0}=1, b_{0}=0$.

The RF pulse is described in polar coordinates $B_{1}(t)=r(t) \exp (i \vartheta(t))$ with RF amplitude $r(t)$, $\mathrm{RF}$ phase $\vartheta(t)$ and imaginary unit $i$. An equidistant time grid $t_{k}=k \tau, k=0, \ldots, N_{t}$ with step size $\tau=T / N_{t}$ is chosen with piecewise constant RF pulse and Gs described by $\left(r_{m}, \vartheta_{m}, g_{m}\right)$, $m=1, \ldots, N_{t}$. The coefficients $a_{m}, b_{m}$ are then given by

$$
\begin{aligned}
\alpha_{m} & =\cos \left(\phi_{m} / 2\right)+i \gamma \tau z g_{m} \sin \left(\phi_{m} / 2\right) / \phi_{m}, \\
\beta_{m} & =i \gamma \tau r_{m} \exp \left(i \vartheta_{m}\right) \sin \left(\phi_{m} / 2\right) / \phi_{m},
\end{aligned}
$$

with $\phi_{m}=-\gamma \tau \sqrt{r_{m}^{2}+\left(z g_{m}\right)^{2}}$ and the gyromagnetic ratio $\gamma$. Here, the variables $a_{m}, b_{m}, \alpha_{m}, \beta_{m}, \phi_{m}$ depend on the spatial coordinate $z \in \Omega=[-L, L]$ in slice direction based on an equidistant spatial discretization $-L=z_{1}<\cdots<z_{N_{z}}=L$ with step size $\delta$.

\subsection{Optimal control of the Bloch equation in the spin-domain}

The slice-selective excitation or refocusing is modeled as optimal control problem with inequality constraints. We jointly optimize for the RF pulse and Gs amplitude, hence we define the control vector $\mathbf{x}=\left(r_{1}, \ldots, r_{N_{t}}, \vartheta_{1}, \ldots, \vartheta_{N_{t}}, g_{2}, \ldots, g_{N_{t}-1}\right)^{T}$. The boundary values for the Gs amplitude are fixed $g_{1}=g_{0}$ and $g_{N_{t}}=g_{T}$ with given $g_{0}, g_{T} \in \mathbb{R}$. The optimal control problem is to minimize the pulse power

$$
\min _{\mathbf{x}} \frac{\tau}{2} \sum_{m=1}^{N_{t}} r_{m}^{2}
$$

subject to the spin domain Bloch equation (1) in every spatial point $z_{j}, j=1, \ldots, N_{z}$, and an amplitude constraint on the Gs slew rate

$$
\left|g_{m}-g_{m-1}\right| \leq \tau s_{\max }, \quad m=2, \ldots, N_{t},
$$

with given $s_{\max }>0$. Additionally, we prescribe amplitude constraints $r(t) \leq r_{\max }$ with $r_{\max }>0$ and bounds for Gs $g_{\min } \leq g(t) \leq g_{\max }$ denoted by $g_{\max }>0$ and $g_{\min }<g_{\max }$. They are collected in the pointwise control constraints

$$
\mathbf{c}_{\min } \leq \mathbf{x} \leq \mathbf{c}_{\max }
$$

with vectorized lower and upper bound $\mathbf{c}_{\min }=\left(0, \ldots, 0,-\pi, \ldots,-\pi, g_{\min }, \ldots, g_{\min }\right)^{T}$ and $\mathbf{c}_{\max }=$ $\left(r_{\max }, \ldots, r_{\max }, \pi, \ldots, \pi, g_{\max }, \ldots, g_{\max }\right)^{T}$.

The slice profile accuracy is now modeled by constraints on the profile and on the phase according to the type of the RF pulse, see [6, Tab 2.3] for excitation, inversion or refocusing pulses. Below, we concentrate on a spin-echo profile for SMS refocusing with initial magnetization $\left(0, M_{0}, 0\right)^{T}$ and ideal crusher gradients. The refocusing profile at the terminal time $T$ is described by $\left|b_{N_{t}}(z)\right|^{2}$ using the last Cayley-Klein parameter $b_{N_{t}}=b_{N_{t}}(z)$ in (1). It is enforced to stay in a neighborhood of the ideal rectangular refocusing for all $z$ in the observation domain $\Omega_{\text {obs }} \subset \Omega$, which is partitioned into the in-slice and out-of-slice domain $\Omega_{\text {obs }}=\Omega_{\text {in }} \cup \Omega_{\text {out }}$.

$$
\begin{aligned}
\left|b_{N_{t}}(z)\right|^{2}-1 \leq e(z), \quad \forall z \in \Omega_{\text {in }}, \\
\left|b_{N_{t}}(z)\right|^{2} \leq e(z), \quad \forall z \in \Omega_{\text {out }},
\end{aligned}
$$


with tolerance $e=e(z)>0$ that may depend on $z$. In particular a different in-slice and out-of-slice error can be prescribed. The profile is not fixed in between the two regions i.e. on $\Omega \backslash\left(\Omega_{\text {in }} \cup \Omega_{\text {out }}\right)$. For ease of notation we do not write the dependence on $z_{j}$ below and introduce the vector $\mathbf{b}=\left(b_{N_{t}}\left(z_{1}\right), \ldots, b_{N_{t}}\left(z_{N_{z}}\right)\right)$. In case of $N_{\mathrm{MB}}$ slices with in-slice domain $S_{l}, l=1, \ldots, N_{\mathrm{MB}}, \Omega_{\mathrm{in}}=\cup_{l=1}^{N_{\mathrm{MB}}} S_{l}$ a constant phase per slice can be modeled as

$$
\left|\varphi(b(z))-\bar{\varphi}_{l}(\mathbf{b})\right| \leq e_{p}, \quad \forall z \in S_{l}, \quad l=1, \ldots, N_{\mathrm{MB}},
$$

with phase $\varphi(b(z))=\arg \left(b_{N_{t}}^{2}(z)\right)$ and arithmetic mean of the phase $\bar{\varphi}_{l}(\mathbf{b})$ in slice $l$, and tolerance $e_{p}=e_{p}(z)>0$.

The control constraints (5) and the state constraints (6), (7) need different solution techniques. Below, we suggest semismooth Newton techniques for the pointwise control constraints (5), which is computationally very inexpensive. In contrast, $(4)$ and the state constraints $(6,7)$ will be treated by an iterative penalization method.

\subsection{Penalization}

State-constrained optimal control problems are known to be challenging since the Lagrange multipliers are typically irregular, which may lead to a decrease of the convergence speed and accuracy of numerical solution methods. To address these difficulties, regularization techniques within Newton-type methods for state-constrained optimal control problems were introduced by several authors, in particular primal-dual active-set strategies applied to a Moreau-Yosida regularization $[4,5]$, which are under appropriate conditions equivalent to a semismooth Newton method [16]. Solution by interior-point methods were proposed in [32]. For further investigations of the topic we refer to $[17]$ and the references therein.

Here we suggest a related but different method, which is designed to facilate the convergence and globalization properties of the problem at hand. We suggest an $L^{p}$-penalization of the state constraints (6) with parameters $\mu_{\text {out }}, \mu_{\text {in }}>0$, and an integer exponent $p \geq 1$, which is driven to $\infty$ as we approach the optimizer. Therefore, we eliminate (6) and add

$$
\frac{\delta \mu_{\mathrm{out}}}{2 p} \sum_{z_{j} \in \Omega_{\mathrm{out}}}\left(\frac{\left|b_{N_{t}}\right|^{2}}{e}\right)^{p}+\frac{\delta \mu_{\mathrm{in}}}{2 p} \sum_{z_{j} \in \Omega_{\mathrm{in}}}\left|\frac{\left|b_{N_{t}}\right|^{2}-1}{e}\right|^{p}
$$

to the objective with parameters $\mu_{\text {in }}, \mu_{\text {out }}>0$ and the spatial step size $\delta>0$. While $p=1$ corresponds to the widespread quadratic tracking, the power- $p$ penalty recovers the original state constraint (6) for $p \rightarrow \infty$. This follows from the simple observation that $|s|^{p} \rightarrow \infty$ for $|s|>1$ and $|s|^{p} \rightarrow 0$ for $|s|<1$ if $p \rightarrow \infty$. We propose a loop around the optimization where $p$ is increased successively until the minimum pulse power solution is attained. We do not start with large $p$ from the beginning, since a small value of $p$ turns out to be advantageous for the globalization, as will be shown in the results section.

The constraints on $g$ in (4) and on the in-slice phase in (7) are treated analogously. For algorithmic purposes we also add a small regularization for the controls $\vartheta_{m}, g_{m}$ with parameter $\zeta>0$, which is driven to 0 as we approach the optimizer. To avoid absolute values we restrict $p>0$ to be even. Altogether the penalized objective is defined as

$$
\begin{gathered}
J(\mathbf{x}, \mathbf{b})=\frac{\tau}{2} \sum_{m=1}^{N_{t}} r_{m}^{2}+\zeta \vartheta_{m}^{2}+\zeta g_{m}^{2}+\frac{\delta \mu_{\mathrm{out}}}{2 p} \sum_{z_{j} \in \Omega_{\mathrm{out}}}\left(\frac{\left|b_{N_{t}}\right|^{2}}{e}\right)^{p} \\
+\frac{\delta \mu_{\mathrm{in}}}{2 p} \sum_{z_{j} \in \Omega_{\mathrm{in}}}\left(\frac{\left|b_{N_{t}}\right|^{2}-1}{e}\right)^{p}+\frac{\delta \mu_{\mathrm{p}}}{p} \sum_{l=1}^{L} \sum_{z_{j} \in S_{l}}\left(\frac{\varphi-\bar{\varphi}_{l}}{e_{p}}\right)^{p}+\frac{\tau \mu_{g}}{p} \sum_{m=2}^{N_{t}}\left(\frac{g_{m}-g_{m-1}}{\tau s_{\max }}\right)^{p}
\end{gathered}
$$

with parameters $\zeta, \mu_{p}, \mu_{g}>0$. 


\section{Methods}

\subsection{Semismooth (quasi-)Newton method}

Semismooth Newton methods are a generalization of Newton's method for specific nonsmooth equations. In the context of optimal control problems they were introduced by $[16,30]$, see also the monographs $[18,31]$. With local superlinear convergence, these methods turned out to be highly efficient for optimal control problems with pointwise control or (regularized) state constraints, with sparsity, or with variational inequalities. Here, the penalized optimal control problem $(1,5,9)$ for a fixed $p$ is solved with semismooth Newton methods. Semismooth Newton methods fulfill the remaining inequality constraints efficiently based on projections. This procedure allows for the inclusion of these constraints into the optimization code without increasing the computational effort. For efficiency the optimization is done purely on the controls $\mathbf{x}$, while the state variables are eliminated using the discrete Bloch and auxiliary equations. We introduce the reduced objective

$$
j(\mathbf{x})=J(\mathbf{x}, \mathbf{b}(\mathbf{x}), \mathbf{w}(\mathbf{x}))=\frac{1}{2} \mathbf{x}^{T} E \mathbf{x}+F(\mathbf{x}),
$$

with the diagonal matrix $E=\tau \operatorname{diag}(1, \ldots, 1, \zeta, \ldots, \zeta, \zeta, \ldots, \zeta)$ and penalization terms $F$. With its gradient $\mathbf{j}^{\prime}(\mathbf{x})=E \mathbf{x}+\mathbf{F}^{\prime}(\mathbf{x})$ the first order necessary optimality conditions for $\min _{\mathbf{c}_{\min } \leq \mathbf{x} \leq \mathbf{c}_{\max }} j(\mathbf{x})$ are given by

$$
\mathbf{x}=P_{\mathrm{ad}}\left(-E^{-1} \mathbf{F}^{\prime}(\mathbf{x})\right)
$$

where $P_{\mathrm{ad}}=\min \left(\mathbf{c}_{\max }, \max \left(\mathbf{c}_{\min }, z\right)\right)$ denotes the componentwise projection to the feasible set. $\mathbf{F}^{\prime}(\mathbf{x})$ is given in terms of a forward and backward solve using a Lagrange calculus in Appendix A. The calculations are done with the Wirtinger calculus [11], which for the spin domain description allows for an efficient derivation and a compact form of both, the equations and the subsequent code. We reformulate (10) equivalently by introducing $\mathbf{c}:=-E^{-1} \mathbf{F}^{\prime}(\mathbf{x})$ as independent variable and parametrize the control $\mathbf{x}=P_{\mathrm{ad}}(\mathbf{c})$. Then a minimizer has to fulfill $\mathrm{G}(\mathbf{c}):=E \mathbf{c}+\mathrm{F}^{\prime}\left(P_{\text {ad }}(\mathbf{c})\right)=0 . \mathrm{G}$ is nonsmooth but semismooth, which allows for the semismooth Newton iteration $\mathbf{c}^{k+1}=\mathbf{c}^{k}+\delta \mathbf{c}$,

$$
D_{N} G\left(\mathbf{c}^{k}\right) \delta \mathbf{c}=-\mathrm{G}\left(\mathbf{c}^{k}\right) .
$$

Therein, $D_{N} G\left(\mathbf{c}^{k}\right) \delta \mathbf{c}$ is the generalized Newton-derivative of $G$ applied to the direction $\delta \mathbf{c}$ in the current point $\mathbf{c}^{k}$. While the assembling of the full matrix $D_{N} G\left(\mathbf{c}^{k}\right)$ is computationally expensive, it is well-known that the evaluation of a matrix vector product can be performed efficiently, without knowledge of the full matrix. This technique is basis of matrix-free Newton-Krylov methods, where the Newton equation is solved iteratively using a Krylov method. We already presented this technique in [1] in the absence of inequality constraints and for the CrankNicolson Bloch solver. Here, we apply the technique in the spin domain, generalize the Newton method to the semismooth case with inequality constraints, and to quasi-Newton methods. By the calculus for Newton derivatives [31], the left-hand side of (11) can be computed as

$$
D_{N} G\left(\mathbf{c}^{k}\right) \delta \mathbf{c}=E \delta \mathbf{c}+F^{\prime \prime}\left(P_{\mathrm{ad}}\left(\mathbf{c}^{k}\right)\right) D_{N} P_{\mathrm{ad}}\left(\mathbf{c}^{k}\right) \delta \mathbf{c}
$$

where $D_{N} P_{\text {ad }}\left(\mathbf{c}^{k}\right)$ is the Newton-derivative of the projection $P_{\text {ad }}$ at the current iterate $\mathbf{c}^{k}$. The second summand can be realized by a forward backward solve consisting in a linearized Bloch equation and its adjoint analogously to [1, eq. $(6,7)]$. We introduce the inactive set $I=\{m \mid-$ $\left.c_{\min , m}<c_{m}<c_{\max , m}\right\}$ and its characteristic function $\chi_{I}(m)$ which is 1 for $m \in \mathcal{I}$ and 0 otherwise. Then it holds that $D_{N} P_{\text {ad }}(\mathbf{c})=\chi$ with $\chi=\operatorname{diag}\left(\chi_{I}(1), \ldots, \chi_{I}\left(3 N_{t}-2\right)\right)$. In order to save computational effort, the system is firstly solved on the inactive set $\mathcal{I}$, where $D_{N} G(\mathbf{c})$ is 
symmetric, using the Steihaug-cg method [29]. Finally the components on the active set can be easily obtained by adding the last residual, for background see [26, Algorithm 2]. Steihaug-cg is embedded into a trust-region framework based on [29] and [26, Algorithm 3].

In case of a semismooth quasi-Newton method we approximate $F^{\prime \prime}$ using the Broyden-Fletcher-GoldfarbShanno (BFGS) formula [23]

$$
B_{k+1}=B_{k}-\frac{B_{k} \mathbf{s}_{k} \mathbf{s}_{k}^{T} B_{k}}{\mathbf{s}_{k}^{T} B_{k} \mathbf{s}_{k}}+\frac{\mathbf{y}_{k} \mathbf{y}_{k}^{T}}{\mathbf{y}_{k}^{T} \mathbf{s}_{k}},
$$

with $\mathbf{s}_{k}=\mathbf{x}^{k+1}-\mathbf{x}^{k}$ and $\mathbf{y}_{k}=\mathbf{F}^{\prime}\left(P_{\mathrm{ad}}\left(\mathbf{c}^{k+1}\right)\right)-\mathbf{F}^{\prime}\left(P_{\mathrm{ad}}\left(\mathbf{c}^{k}\right)\right)$. The expression $D_{N} G(\mathbf{c}) \delta \mathbf{c}$ is then replaced by $\left(E+B_{k+1} \chi\right) \delta \mathbf{c}$. The update is skipped if the trust-region step is rejected. For efficiency, we apply $B_{k+1}$ in the (matrix-free) limited-memory BFGS method using the compact form of [23] and [7], which requires less storage and computational effort by storing only data from the last $L_{\mathrm{BFGS}}$ steps with a fixed limit $L_{\mathrm{BFGS}} \in \mathbb{N}$.

We note that the presented trust-region semismooth Newton method coincides with the trustregion Newton method of [1] in the absence of control constraints (5). In this case $P_{\mathrm{ad}}(\mathbf{c})=\mathbf{c}$, and the active set is empty.

\subsection{Implementation}

The optimal control approach is implemented in MATLAB (The MathWorks, Inc., Natick, USA). The Bloch state and adjoint solvers are parallelized in $\mathrm{C}$ using OpenMP and included using a MEX file. The computations are done on one node of the HPC Cluster "RADON 1" (RICAM Linz, Austria) with $16 \mathrm{CPU}$ cores with $2.4 \mathrm{GHz}$.

The parameters of the penalized objective are adapted automatically throughout the optimization. Therefore, the maximum errors in the constraints (4), (6), (7) are defined

$$
\begin{aligned}
\varepsilon_{g} & =\max _{m=2, \ldots, N_{t}}\left|g_{m-1}-g_{m}\right| /\left(\tau s_{\max }\right), \\
\varepsilon_{\text {out }} & =\max _{z \in \Omega_{\text {out }}}\left|b_{N_{t}}\right|^{2} / e \\
\varepsilon_{\text {in }} & =\max _{z \in \Omega_{\text {in }}}\left(1-\left|b_{N_{t}}\right|^{2}\right) / e \\
\varepsilon_{\mathrm{ph}} & =\max _{l=1, \ldots, L} \max _{z \in S_{l}}\left|\varphi-\bar{\varphi}_{l}\right| / e_{p} .
\end{aligned}
$$

Note that these errors are dimensionless and scaled to 1 . Every 20th optimization step we adapt the parameter $\mu_{g}$ by multiplication with $\min \left(10, \max \left(0.3,1+10\left(\varepsilon_{g}-1\right)\right)\right)$. Accordingly we increase $\mu_{g}$ if $g$ is not admissible to the slew rate constraint $\left(\varepsilon_{g}>1\right)$, keep $\mu_{g}$ if $g$ is admissible but active $\left(\varepsilon_{g}=1\right)$, and reduce it if the slew rate constraint is not active at all $\left(\varepsilon_{g}<1\right)$. The other parameters $\left(\mu_{\text {out }}, \mu_{\text {in }}, \mu_{p}\right)$ are adapted in the same way by exchanging $\varepsilon_{g}$ with $\varepsilon_{\text {out }}, \varepsilon_{\text {in }}, \varepsilon_{\mathrm{ph}}$. This technique ensures that the different penalty terms remain balanced, and that the results are insensitive to the initialization of the parameters. Initially we set $\mu_{\text {out }}=10^{5}, \mu_{\text {in }}=10^{4}, \mu_{\mathrm{p}}=1$, and $\mu_{\mathrm{g}}=1$.

\section{Results}

In this section we demonstrate the application of the proposed design method to reduce the overall RF pulse power using different initial guesses in the field of SMS refocusing. We consider four different experiments for a varying number of slices and slice thicknesses. The initial pulses are designed with six different state of the art methods for SMS RF pulse design [3] 
Table 1: Parameters of the experiments. tr denotes the transition region

\begin{tabular}{ccccccccccc}
\hline Example & MB factor & $\begin{array}{c}T \\
\mathrm{~ms}\end{array}$ & $N_{t}$ & $\begin{array}{c}L \\
\mathrm{~mm}\end{array}$ & $N_{z}$ & $\begin{array}{c}\mathrm{tr} \\
\mathrm{mm}\end{array}$ & $\begin{array}{c}s_{\max } \\
\mathrm{T} \mathrm{m}^{-1} \mathrm{~s}^{-1}\end{array}$ & $\left.e\right|_{\Omega_{\text {in }}}$ & $\left.e\right|_{\Omega_{\text {out }}}$ & $e_{p}$ \\
\hline SUP MB3 & 3 & 10.42 & 515 & 60 & 2401 & 1.1 & 200 & 0.03 & 0.02 & $\infty$ \\
Root-flipped MB6 & 6 & 8.41 & 258 & 36 & 961 & 1.2 & 200 & 0.05 & 0.04 & $\infty$ \\
PINS MB10 & 10 & 9.46 & 946 & 120 & 4801 & 1.2 & 200 & 0.03 & 0.02 & 0.01 \\
Comparison MB5 & 5 & 6.02 & 602 & 125 & 5001 & 1.8 & 200 & 0.03 & 0.02 & 0.01 \\
\hline
\end{tabular}

including a conventional superposition [22], superposition with VERSE [9, 15], PINS [24], PINS with VERSE, MultiPINS [10] and root-flipped SMS design [28]. For all simulations we assume perfect spoiling for the computed refocusing profile in the spin-domain description [25]. Tab. 1 lists the parameters of the four examples. The peak amplitudes are generally set to $r_{\max }=18$ $\mu \mathrm{T}$ for $B_{1}, g_{\max }=24 \mathrm{mT} \mathrm{m}^{-1}$ for the gradient, and $200 \mathrm{~T} \mathrm{~m}^{-1} \mathrm{~s}^{-1}$ for the gradient slew rate. The minimum gradient value $g_{\min }$ is set to $-g_{\max }$. However, the second example below investigates the influence of the choice of $s_{\max }$ and $g_{\min }$ on the optimal solution. Typical spatial and the temporal discretizations are chosen (50 to $75 \mu \mathrm{m}$ and 10 to $40 \mu \mathrm{s}$ ). The excluded transition regions are listed in Tab. 1 and shown graphically in Row 3 of Fig. 1-5 where black lines mark the in-slice/out-of-slice error tolerance. In all examples we set $g_{T}=g_{0}$ determined by the used initial guess. To compare the overall pulse power of the initials and the optimized RF pulse candidates, we compute the (scaled) pulse power using

$$
S=10^{4} \tau \sum_{m=1}^{N_{t}} r_{m}^{2}, \quad[S]=10^{-5} \mathrm{~T}^{2} \mathrm{~s} .
$$

As standard parameters for the optimization the exponent $p$ in the penalization is initially set to 2 and increased by a factor of 2 every 350th iteration of the quasi-Newton method (with $L_{\mathrm{BFGS}}=30$ ), which is shown below to be the preferable strategy in general. In addition, we give the best results using individually tuned parameters per example. Whenever $p$ is increased, $\zeta$ is divided by 10 starting from $\zeta=0.01$.

\subsection{Superposition (SUP) MB3}

We designed a 180 degree single slice refocusing pulse based on the SLR [25] (in-slice and out-of-slice error of 0.02 , time bandwidth product (TBP) $=2.8$ and slice thickness of $2 \mathrm{~mm}$ ). To generate a SMS refocusing pulse with a MB factor of three, we superposed three partially phase shifted subpulses together with a constant Gs amplitude. The symmetry of the slices around the isocenter leads to a real valued pulse, rather than a complex pulse. This RF pulse was scaled to a maximal peak $B_{1}$ amplitude of $18 \mu \mathrm{T}$ resulting in a pulse duration of $10.42 \mathrm{~ms}$ and is shown, together with a Bloch simulation, in the first row of Figure 1. The optimized results for this example are given in the second row of Figure 1. The simulated refocusing profile is shown for the full field of view (FOV) of $120 \mathrm{~mm}$ and in detail for the central slice in the third row of Figure 1 with the non-optimized but still stable phase. The overall pulse power is reduced by $59 \%$ from initially $S=1.859$ to $S=0.767$ after the optimization.

Different strategies for choosing the penalty exponent $p$ were tested, see Tab. 2. In the upper seven cases 3500 quasi-Newton iterations are performed in total. The optimized values of $S$ for the first three examples presented in this paper are depicted. As can be seen in the first three rows, constant values of $p$ do not allow to find a small pulse power. Especially, the classical quadratic tracking in the first row cannot improve $S$ within 3500 iterations. 

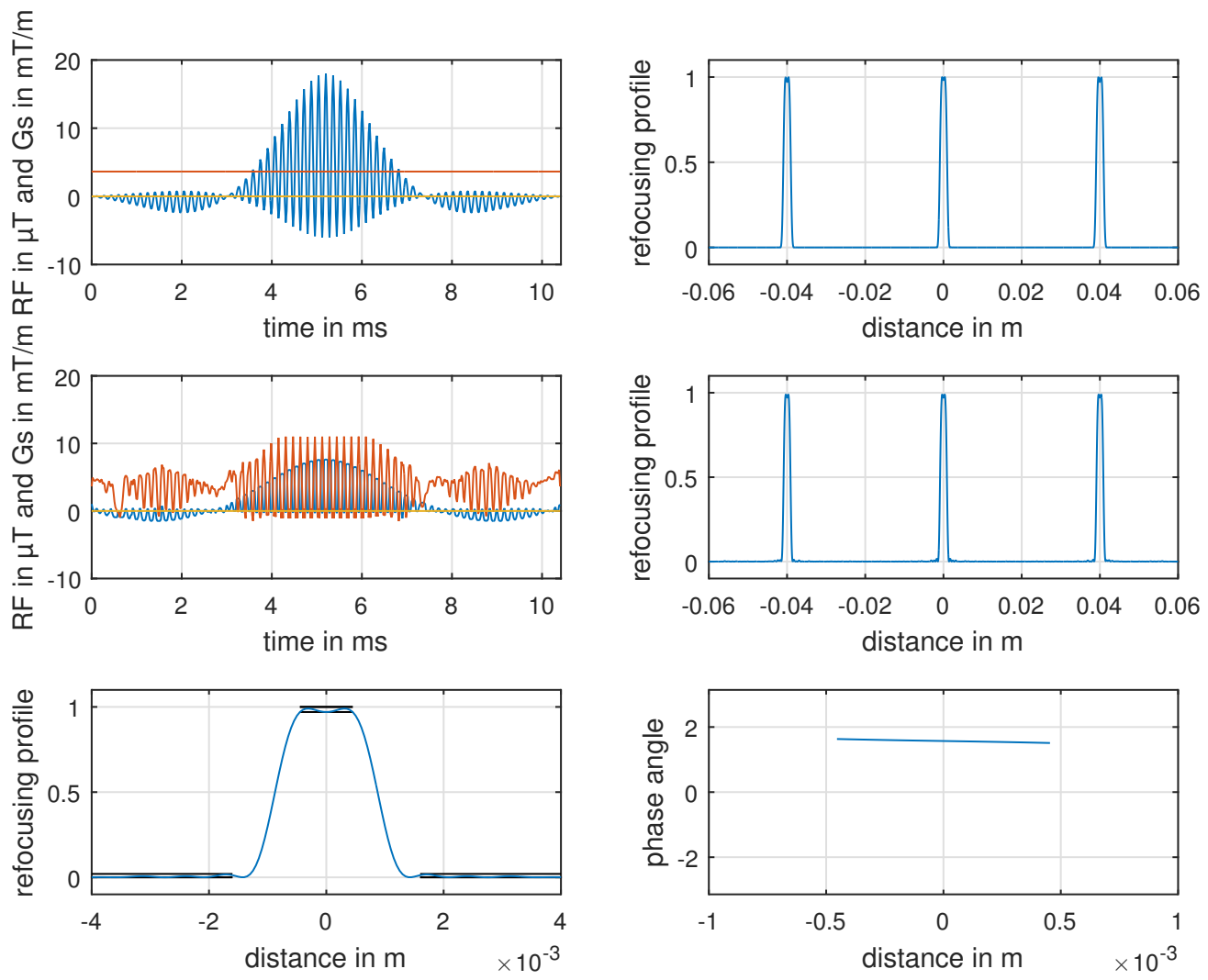

Figure 1: Optimization results and spin domain Bloch simulations for a MB3 pulse. Initial (Row 1) and optimized (Row 2) RF and Gs (Re(RF) in blue, $\operatorname{Im}(\mathrm{RF})$ in yellow and Gs in red) with refocusing profile $\left|b_{N_{t}}\right|^{2}$. Zoom of the central slice (black lines mark the in-slice/outof-slice error tolerance) with phase angle $\left(b_{N_{t}}\right)$ (not part of the optimization) for the optimized pulse (Row 3).

Table 2: Optimization results: optimized pulse power in dependence of the choice rule for $p$ (in MATLAB colon notation). $p$ is increased every maxit iteration (or stopping in case of $\operatorname{maximum} p$ )

\begin{tabular}{cc|ccc}
\hline $\begin{array}{c}\text { choice rule } \\
\text { for } p\end{array}$ & maxit & \multicolumn{3}{|c}{ optimized pulse power $S$} \\
\hline 2 & 3500 & 1.859 & 4.501 & 1.697 \\
$2^{5}$ & 3500 & 0.931 & 3.087 & 1.087 \\
$2^{10}$ & 3500 & 1.298 & 4.501 & 0.929 \\
$2^{1: 10}$ & 350 & 0.767 & 2.199 & 0.819 \\
$4^{1: 5}$ & 700 & 0.768 & 2.240 & 0.828 \\
$2^{4: 10}$ & 500 & 0.770 & 2.232 & 0.851 \\
$8^{1: 4}$ & 875 & 0.799 & 2.433 & 0.879 \\
\hline $2^{1: 10}$ & 100 & 1.577 & 2.632 & 1.697 \\
$2^{1: 10}$ & 250 & 0.771 & 2.237 & 0.840 \\
$2^{1: 10}$ & 350 & 0.767 & 2.199 & 0.819 \\
$2^{1: 10}$ & 450 & 0.797 & 2.184 & 0.816 \\
$2^{1: 10}$ & 500 & 0.805 & 2.168 & 0.914 \\
\hline
\end{tabular}



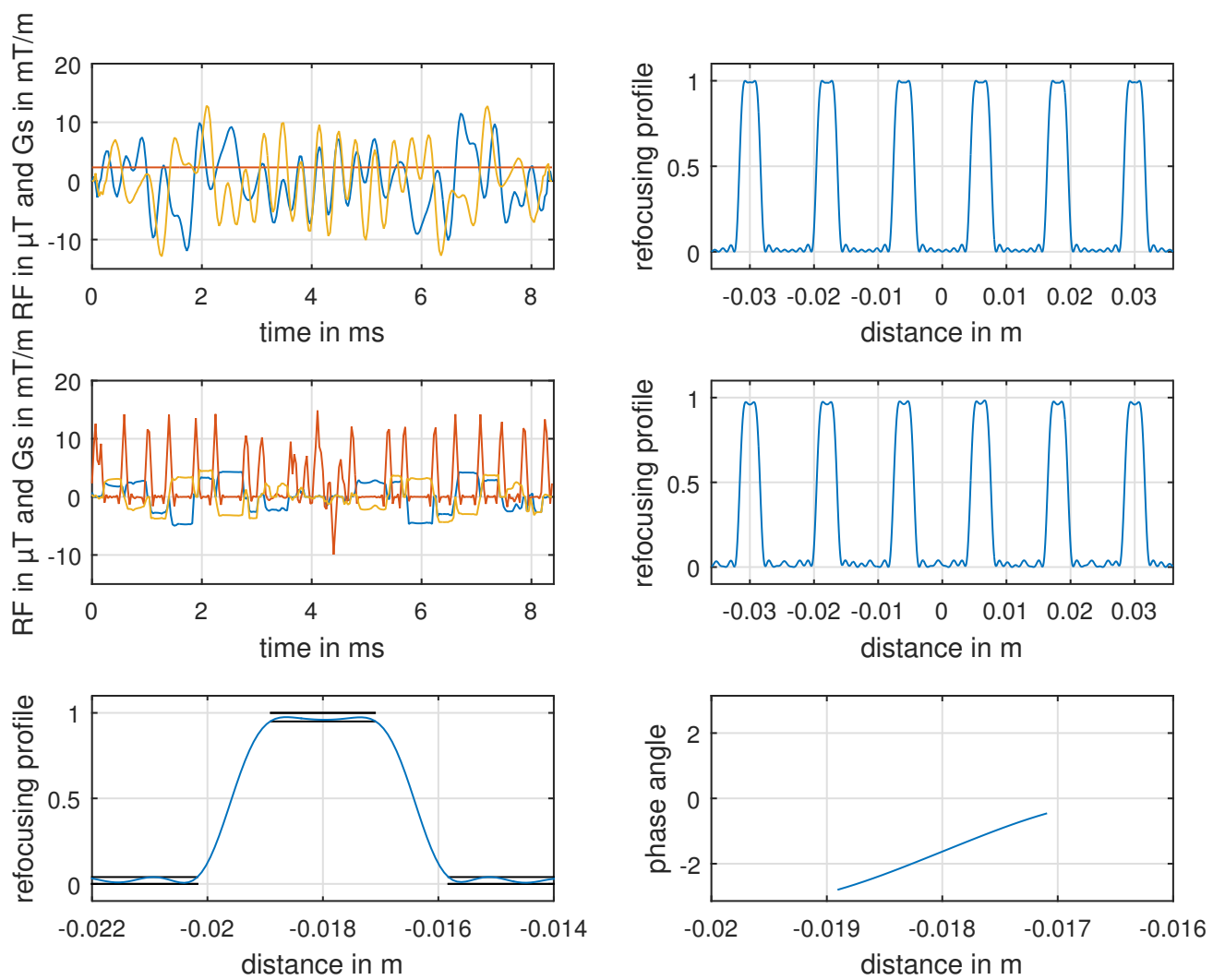

Figure 2: Optimization results and spin domain Bloch simulations for a MB6 pulse. Initial (Row 1) and optimized (Row 2) RF and Gs (Re(RF) in blue, $\operatorname{Im}(\mathrm{RF})$ in yellow and Gs in red) with refocusing profile $\left|b_{N_{t}}\right|^{2}$. Zoom of the central slice (black lines mark the in-slice/outof-slice error tolerance) with phase angle $\left(b_{N_{t}}\right)$ (not part of the optimization) for the optimized pulse (Row 3)

In contrast, the proposed strategy of increasing $p$ from a small initial value to a large value performs well, independently of the particular protocol, see rows four to seven. The best results were gained with the strategy $p=2^{k}, k=1, \ldots, 10$. The lower part shows that the number of 350 iterations is a reasonable choice for that strategy. Therefore, this strategy is applied for all other optimization results throughout the paper. We observed that larger values $p>1024$ lead to many more iterations without a significant gain. However, we mention the best observed run for this example, which yielded a power $S=0.744$ with individually tuned parameters $p=2^{k}, k=1, \ldots, 12$ with maxit $=500$ and $L_{\mathrm{BFGS}}=180$.

\subsection{Root-flipped MB6}

For this example we used a SMS refocusing pulse designed with the root-flipped method [28] as an initial guess for the numerical optimization together with a constant Gs amplitude. This complex RF pulse refocuses six slices with a slice thickness of $1.75 \mathrm{~mm}$ and $\mathrm{TBP}=4$. Scaled to a peak $B_{1}$ amplitude of $13 \mu \mathrm{T}$, the pulse duration results in $9.46 \mathrm{~ms}$ with $S=4.501$. By optimization the pulse power was reduced down to approximately $S=2.2$ corresponding to a SAR reduction of $51 \%$. Below we show that the reduction can even be increased to $77 \%$. This best solution is plotted together with the initial guess in Figure 2. Bloch simulations at the terminal time of the spoiled refocusing profile are given for the full $F O V=72 \mathrm{~mm}$ and in detail for one slice together with the refocusing phase. Please note, that the phase was intentionally not part of the optimization. 
Table 3: Optimization results: optimal pulse power in dependence of the optimization method. maxit is the iteration per step for $p$, i.p. denotes individually tuned parameters per example.

\begin{tabular}{lcccc}
\hline optimization method & maxit & \multicolumn{3}{c}{ optimized pulse power $S$} \\
& & SUP & RF & PINS \\
& & MB3 & MB6 & MB10 \\
\hline semism. Newton, $p=2^{2: 7}$ & 300 & & 1.471 & \\
semism. Newton & i.p. & & 1.041 & \\
semism. BFGS, $L_{\mathrm{BFGS}}=10$ & 350 & 0.790 & 2.257 & 0.997 \\
semism. BFGS, $L_{\mathrm{BFGS}}=30$ & 350 & 0.767 & 2.199 & 0.819 \\
semism. BFGS, $L_{\mathrm{BFGS}}=50$ & 350 & 0.763 & 2.172 & 0.813 \\
semism. BFGS, $L_{\mathrm{BFGS}}=80$ & 350 & 0.761 & 2.163 & 0.808 \\
semism. BFGS, $L_{\mathrm{BFGS}}=200$ & i.p. & 0.744 & 1.166 & 0.803 \\
\hline
\end{tabular}

Table 4: Optimization results for a MB6 pulse: optimal pulse power $S$ in dependence of the maximum slew rate $s_{\max }$ and the minimum gradient field $g_{\min }$.

\begin{tabular}{cccc}
\hline$s_{\max }$ & $g_{\min }$ & optimized $S$ & SAR reduction \\
\hline 200 & -24 & 2.199 & $51.1 \%$ \\
100 & -24 & 2.248 & $50.1 \%$ \\
50 & -24 & 2.357 & $47.6 \%$ \\
30 & -24 & 2.458 & $45.4 \%$ \\
10 & -24 & 2.683 & $40.4 \%$ \\
\hline 200 & -24 & 2.199 & $51.1 \%$ \\
200 & 0 & 2.211 & $50.9 \%$ \\
200 & 1 & 2.289 & $49.1 \%$ \\
200 & 2 & 2.621 & $41.8 \%$ \\
200 & 2.3 & 2.896 & $35.7 \%$ \\
200 & 2.3091 & 2.901 & $35.6 \%$ \\
\hline
\end{tabular}

Here, we compare different values of the limit parameter $L_{\mathrm{BFGS}}$ for the limit-memory BFGS, as well as the full semismooth Newton method, see Tab. 3. As can be seen from the table, $L_{\mathrm{BFGS}}=10$ does not improve the pulse power satisfactorily. In contrast, $L_{\mathrm{BFGS}}=30$ already yields very small values in a computation time of $2.3 \mathrm{~min}$. Further increasing the limit in the BFGS method yields slightly better results at the costs of a higher computational effort. Due to this trade-off we decided to use $L_{\mathrm{BFGS}}=30$ in all other optimization results. However, if much more computational time can be invested, the SAR of the MB6 pulse can be even reduced by $74 \%$ using individually tuned parameters. Then, the pulse power is reduced to $S=1.166$ using $p=2^{k}, k=1, \ldots, 10$ with maxit $=5000$ and $L_{\mathrm{BFGS}}=200$ leading to a computation time of 55 min. On the other hand, the exact Hessian within the semismooth Newton method allows for the best power values that were observed at the cost of an increased numerical effort. Here, we find $S=1.471$ in the standard configuration (300 iterations per step for $p=2^{k}, k=2, \ldots, 7$ ) with a computation time of about $2 \mathrm{~h}$ (with standard MATLAB parallelization instead of C/OpenMP). With individually tuned parameters the semismooth Newton method gives $S=1.041$ which is a reduction of $77 \%$ of the pulse power.

The influences of the maximum slew rate $s_{\max }$ and the minimum gradient $g_{\min }$ on the gain of the optimal solution are depicted in Tab. 4. With smaller $s_{\max }$ the SAR reduction decreases slightly from $51 \%$ down to $40 \%$. An increase of $g_{\min }$ up to 1 does not influence the optimal SAR significantly, which is related to the given boundary conditions $g_{0}=g_{T}=2.31$. However, larger values decrease the SAR reduction significantly. The optimal solutions for different $s_{\max }$ 
are depicted in Fig. 3. It can be seen that the slew rate is hitting the constraint at many time instances and in all settings. With smaller $s_{\max }$ the gradient waveform gets smoother, while the maximum RF amplitude increases slightly. All optimal solutions show a good slice profile, one that is admissible to the underlying inequality constraints. Up to now the lower constraint on $g$ was never active due to $g_{\min }=-24$. In contrast, Figure 4 shows the optimized results with different tight constraints on the minimal gradient amplitude $\left(\{0,1,2.31\} \mathrm{mT} \mathrm{m}^{-1}\right)$ and standard parameters. As can be seen, the lower bound constraint on $g$ is active at many time instances, especially for $g_{\min }=2.31$.
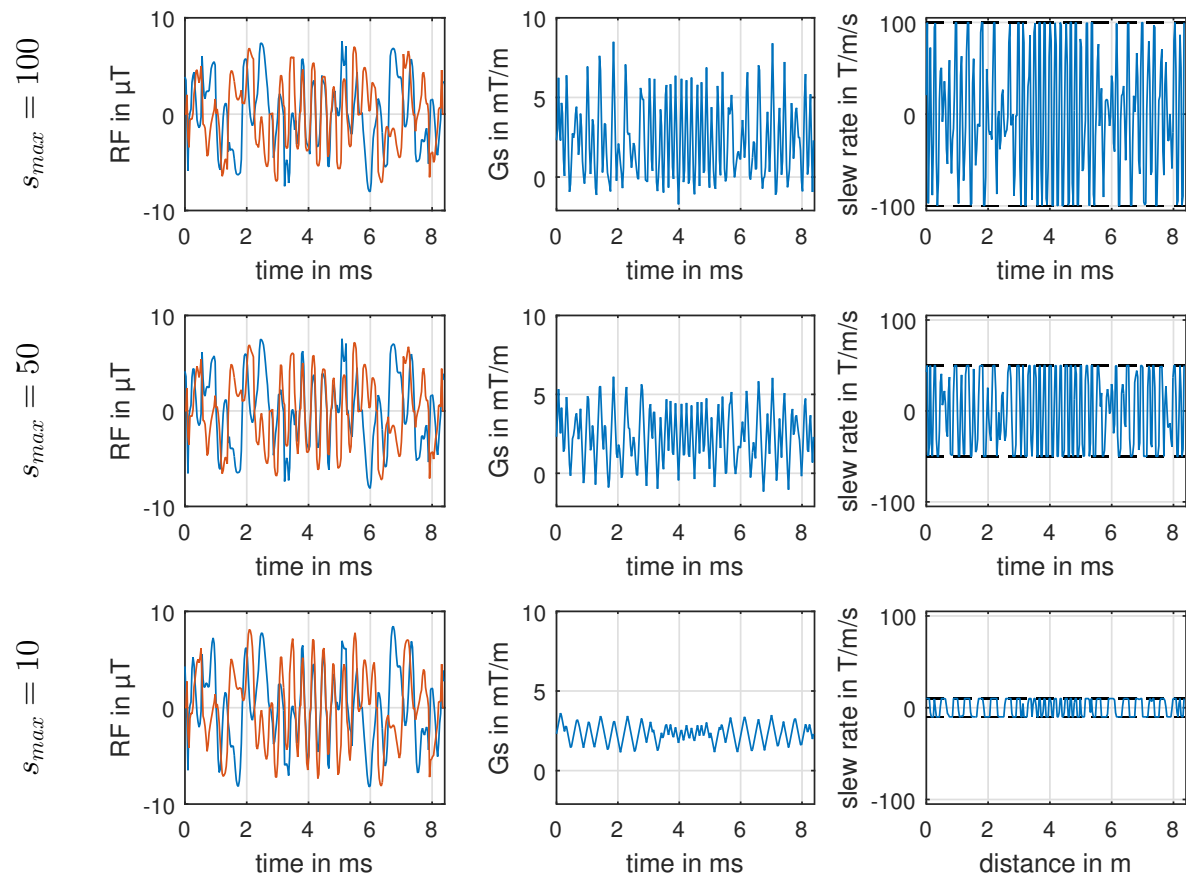

Figure 3: Optimized MB6 RF pulse ( $R e(R F)$ in blue, $\operatorname{Im}(R F)$ in red), slice-selective gradient Gs and gradient slew rate for different maximum values of $s: s_{\max } \in\{100,50,10\} \mathrm{T} \mathrm{m}^{-1} \mathrm{~s}^{-1}$.

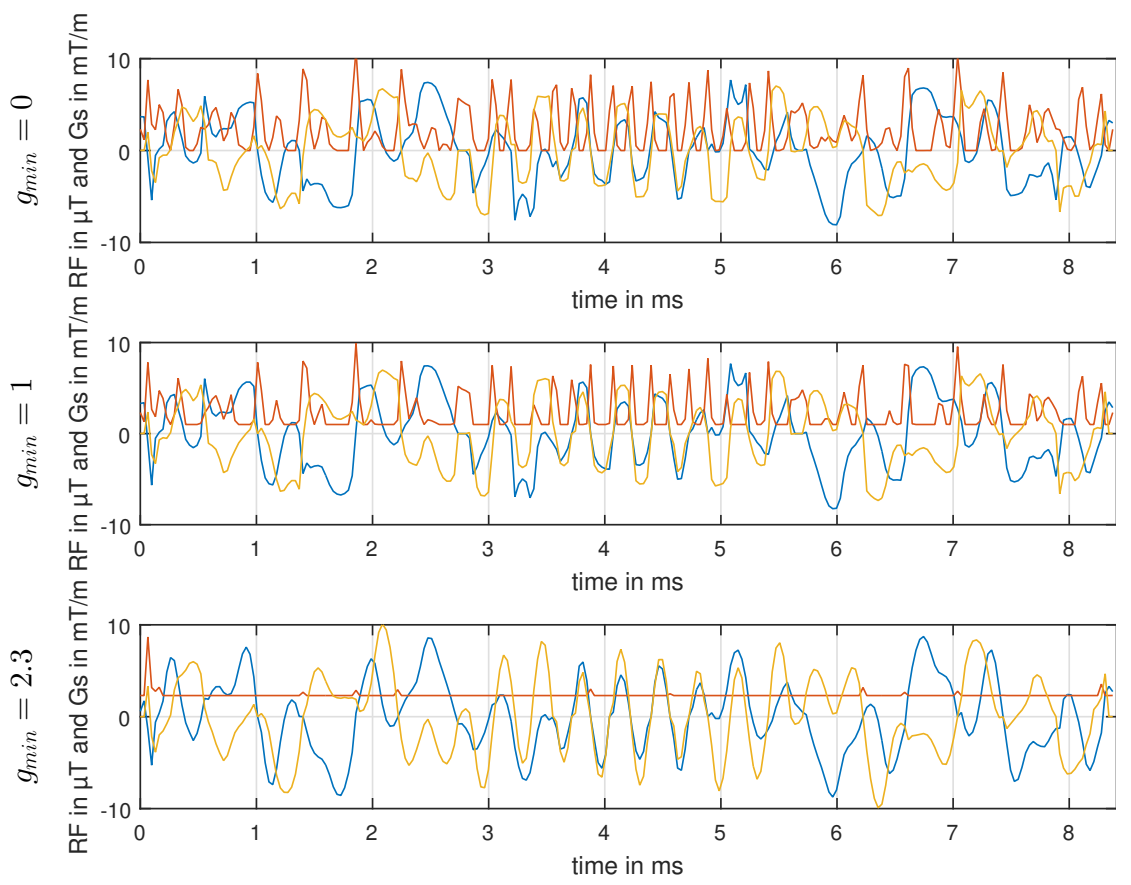

Figure 4: Optimized MB6 RF pulse and slice-selective gradient shape (Re(RF) in blue, $\operatorname{Im}(R F)$ in yellow and Gs in red) for different minimal values of Gs: $g_{\min } \in\{0,1,2.3\} \mathrm{mT} \mathrm{m}^{-1}$. 

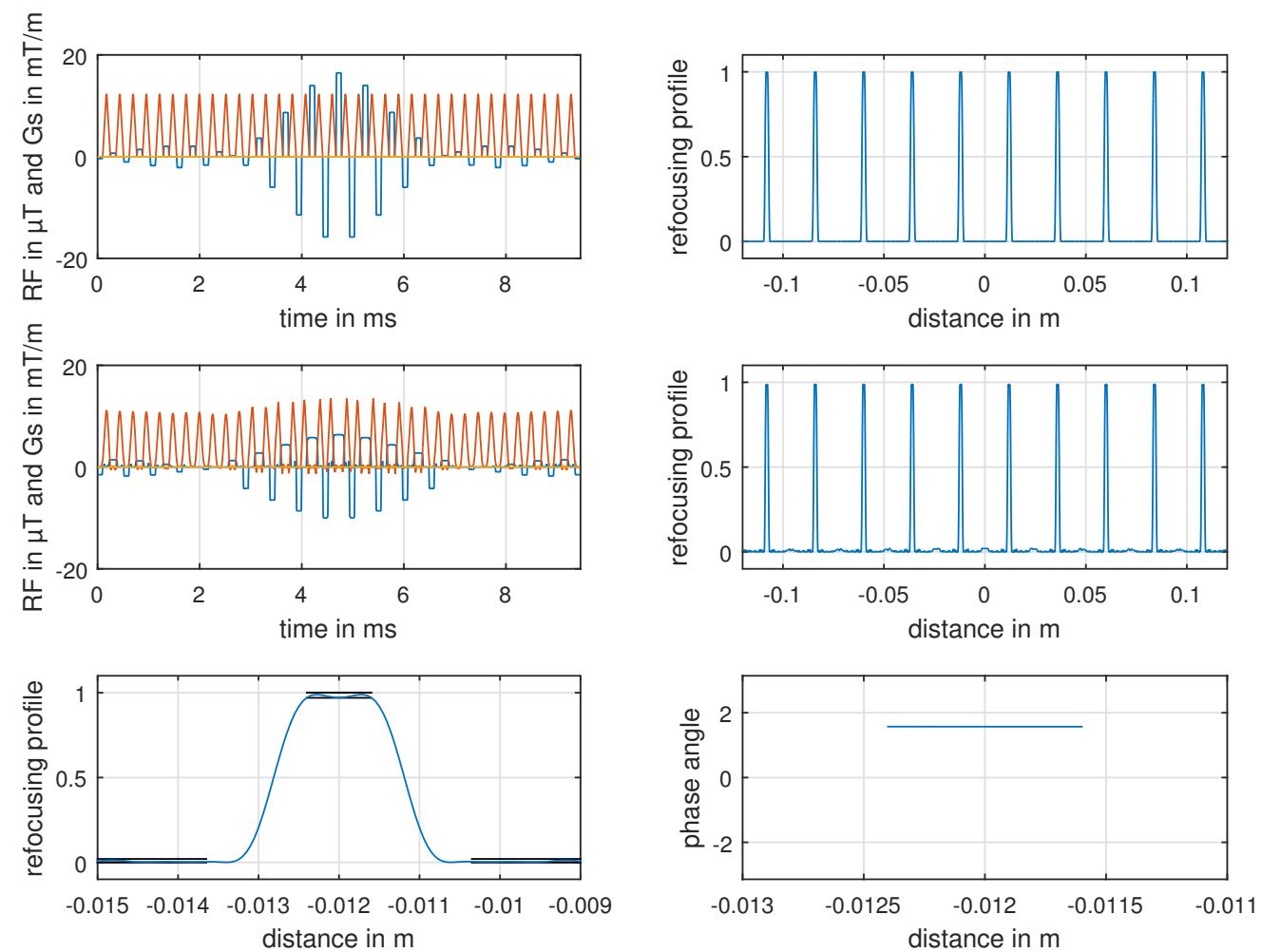

Figure 5: Optimization results and spin domain Bloch simulations for a MB10 pulse. Initial (Row 1) and optimized (Row 2) RF and gradient waveform (Re(RF) in blue, $\operatorname{Im}(\mathrm{RF})$ in yellow and Gs in red) with refocusing profile $\left|b_{N_{t}}\right|^{2}$. Zoom of the central slice (black lines mark the in-slice/out-of-slice error tolerance) with phase angle $\left(b_{N_{t}}\right)$ for the optimized pulse (Row 3)

\subsection{PINS MB10}

For the third example we designed a SMS refocusing pulse applying the PINS method [24] on a SLR based 180 degree refocusing pulse (in-slice and out-of-slice error of $0.02, \mathrm{TBP}=3$, slice thickness of $2 \mathrm{~mm}$ ). Accordingly, the Gs amplitude is built up of regular PINS blips, see the upper left plot of Figure 5. Here, we include phase constraints with a maximum phase deviation of $e_{p}=0.01 \mathrm{rad}$ from the mean phase per slice. This PINS refocusing pulse was scaled to a peak $B_{1}$ amplitude of $18 \mu \mathrm{T}$ producing $T=10.36 \mathrm{~ms}$. The optimized real valued controls are given in Figure 5 together with the used initial and Bloch simulations with $F O V=240 \mathrm{~mm}$.

The power of the shown RF pulse was reduced to $S=0.819$ by roughly $53 \%$ compared to the inital PINS guess. With individually tuned parameters we even find $S=0.803$ with $p=2^{k}, k=$ $1, \ldots, 12$, maxit $=500$ and $L_{\mathrm{BFGS}}=170$. For each example the reduction of the required pulse power is depicted in Tab. 5. The last three rows of Tab. 5 contain the optimization results without joint control, i.e. when only the RF pulse is controlled while $g$ is kept fix at its initialization. For the superposition pulse with a fixed and constant $g$ the reduction in the pulse power $S$ is limited, while for the root-flipped pulse we observe a good reduction of $35 \%$ (compared to $51 \%$ reduction for joint control). In case of PINS initialization, keeping the non-constant $g$ fixed yields a reduction of $46 \%$ which is just slightly below the value of $53 \%$ that was observed for joint control. 
Table 5: Pulse power $S$ of initial and optimized pulse with standard parameters and with individually tuned parameters/method.

\begin{tabular}{cccc}
\hline Example & initial $S$ & optimized $S$ & SAR reduction \\
\hline PINS & 1.724 & 0.819 & $52.5 \%$ \\
PINS best & 1.724 & 0.803 & $53.4 \%$ \\
SUP & 1.859 & 0.767 & $58.7 \%$ \\
SUP best & 1.859 & 0.744 & $60.0 \%$ \\
Root-flipped & 4.501 & 2.199 & $51.1 \%$ \\
Root-flipped best & 4.501 & 1.041 & $76.9 \%$ \\
\hline PINS $(g$ fixed) & 1.724 & 0.925 & $46.3 \%$ \\
SUP $(g$ fixed $)$ & 1.859 & 1.471 & $20.9 \%$ \\
Root-flipped $(g$ fixed) & 4.501 & 2.927 & $35.0 \%$ \\
\hline
\end{tabular}

\subsection{Comparison of different initializations for MB5}

In a final study we compare the performance of the optimization method for different initializations (superposition, superposition with VERSE, PINS, PINS with VERSE, and MultiPINS). For an intermediate MB factor of 5 , these five initializations can be designed with the same duration $(T=6.02 \mathrm{~ms})$ and comparable slice profile properties (slice thickness $2 \mathrm{~mm}, \mathrm{TBP}=2$, maximum in-slice and out-of-slice error, and maximum phase error, see Tab.1) resulting in different peak $B_{1}$ amplitudes and RF power (see Tab.6). The boundary conditions are in general set to $g_{0}=g_{T}=0$, which is implemented with a ramp-up/ramp down for the superposition pulse to reach the constant gradient of $4.65 \mathrm{mT} \mathrm{m}^{-1}$, respectively $5.00 \mathrm{mT} \mathrm{m}^{-1}$ with VERSE. Furthermore, the peak $B_{1}$ for the superposition pulse is projected down to the allowed maximum of $18 \mu \mathrm{T}$. The optimized pulse power $S$ is shown in the last two columns of Tab.6 for standard parameters in the optimization (column $S$ ) and for $L_{\mathrm{BFGS}}=200, p=2^{k}, k=1, \ldots, 12$ with each 350 iterations (column $S$ best). Depicted are also the maximum errors of the slice profile.

In the first two rows the superposition pulse without and with VERSE are compared. We see that the version with VERSE allows for a smaller pulse power $S$ after optimization. The last three rows show that the optimization method yields the best power values for PINS, PINS with VERSE and MultiPINS pulses. While their initial values for $S$ are different, the optimized values in the last column coincide for these three initialization. However, the optimized pulses behind these power values are very different. The maximum errors in the optimum are increased out-of-slice and in the phase, however, the maximum allowed errors are in most cases not attained. The maximum in-slice errors of the initializations and the optima agree and are at the bound 0.03.

\subsection{Experimental validation}

Figure 6 contains the experimental validation of the optimized refocusing pulse and Gs shape for $\mathrm{MB}_{3}$ using a superposition initial (Figure 1), $\mathrm{MB}_{5}$ using a MultiPINS initial (not shown) and MB1o using a PINS initial (Figure 5), on a 3T MR scanner (Magnetom Skyra, Siemens Healthcare, Erlangen, Germany). The data is acquired with a $2 \mathrm{D}$ spin echo sequence ( $\mathrm{TE}=$ $25-30 \mathrm{~ms}, \mathrm{TR}=100 \mathrm{~ms}, \mathrm{FOV}=300 \mathrm{~mm} \times 300 \mathrm{~mm}$, matrix $=1536$ pixel $\times 1536$ pixel $(922$ phase encoding steps), readout bandwidth $=130 \mathrm{~Hz} /$ pixel) with conventional superposed SMS excitation pulses using a cylindrical phantom. Figure 7 contains a zoomed view to one slice of the experimental phantom data shown in Figure 6 and compares it to numerical Bloch simulations of the optimized refocusing pulses. Figure 8 shows the in-vivo reconstruction of the measured slice profiles (optimized MB3) as described above with a TR $=200 \mathrm{~ms}$ and a matrix of 512 pixel $\times$ 512 pixel with a measured head SAR of $0.288 \mathrm{~W} \mathrm{~kg}^{-1}\left(0.512 \mathrm{~W} \mathrm{~kg}^{-1}\right.$ for the initial MB3 pulse). 
Table 6: Performance of the optimization method for different initializations for an MB5 example. Depicted are the maximum errors and the pulse power $S$ both for the initial and optimized pulse. The maximum errors were constrained by 0.03 in-slice, by 0.02 out-of-slice, and by 0.01 in the phase constraint.

\begin{tabular}{|c|c|c|c|c|c|}
\hline \multirow[b]{2}{*}{ Initialization } & \multicolumn{4}{|c|}{ initial } & \multirow[b]{2}{*}{$\begin{array}{c}S \\
10^{-5} \mathrm{~T}^{2} \mathrm{~s}\end{array}$} \\
\hline & $\begin{array}{c}\text { error } \\
\text { in-slice }\end{array}$ & $\begin{array}{c}\text { error } \\
\text { out-of-slice }\end{array}$ & $\begin{array}{l}\text { error } \\
\text { phase }\end{array}$ & $\begin{array}{c}\max \left|B_{1}\right| \\
\mu \mathrm{T}\end{array}$ & \\
\hline SUP & 0.0318 & 0.0142 & 0.0031 & 18 & 1.9531 \\
\hline SUP VERSE & 0.0297 & 0.0016 & 0.0010 & 14 & 1.4461 \\
\hline PINS & 0.0294 & 0.0010 & 0.0009 & 13 & 1.1301 \\
\hline PINS VERSE & 0.0297 & 0.0014 & 0.0008 & 13 & 0.9967 \\
\hline \multirow[t]{2}{*}{ MultiPINS } & 0.0297 & 0.0016 & 0.0009 & 8 & 0.7262 \\
\hline & \multicolumn{4}{|c|}{ optimized } & \\
\hline Initialization & $\begin{array}{c}\text { error } \\
\text { in-slice }\end{array}$ & $\begin{array}{c}\text { error } \\
\text { out-of-slice }\end{array}$ & $\begin{array}{l}\text { error } \\
\text { phase }\end{array}$ & $\begin{array}{c}S \\
10^{-5} \mathrm{~T}^{2} \mathrm{~s}\end{array}$ & $\begin{array}{c}S \text { best } \\
10^{-5} \mathrm{~T}^{2} \mathrm{~s}\end{array}$ \\
\hline SUP & 0.0300 & 0.0066 & 0.0100 & 0.7712 & 0.7677 \\
\hline SUP VERSE & 0.0300 & 0.0200 & 0.0032 & 0.5603 & 0.5556 \\
\hline PINS & 0.0300 & 0.0116 & 0.0030 & 0.4163 & 0.4108 \\
\hline PINS VERSE & 0.0300 & 0.0146 & 0.0093 & 0.4578 & 0.4093 \\
\hline MultiPINS & 0.0300 & 0.0200 & 0.0032 & 0.4144 & 0.4127 \\
\hline
\end{tabular}

\section{Discussion}

We presented a general framework for optimal control based joint design of RF pulses and gradient waveforms for MRI. The framework is flexible in the primary optimization goal, which was chosen as SAR reduction in the examples above, and in the desired magnetization profile. The latter can range from a conventional single slice in the isocenter, to asymmetric or offresonance profiles, or SMS. While we included the most important inequality constraints for RF pulse design, it is possible to add other constraints and treat them with the presented penalization method. Otherwise, constraints can also be turned off by choosing an infinite bound.

The examples were chosen in the context of SMS imaging, where the pulse power and peak $B_{1}$ amplitude are easily exceeded and complying the hardware constraints is crucial for practical applications. Therefore, the optimal control method was tested in the numerical experiments focusing on SAR reduction of several SMS refocusing pulses for various slice thicknesses, time bandwidth products and a slice acceleration factor ranging from MB3 to MB10. The initial pulses are designed with different methods, with conventional superposition [22], VERSE [9, 15], root flipping [28], PINS [24] and MultiPINS [10] with SLR based subpulses [25]. We investigated four different examples for low (MB3), intermediate (MB5 and MB6) and large (MB10) slice acceleration factors. PINS pulses for low MB factors and superposition pulses for large MB factors typically have a very long pulse duration. Therefore we chose to use MB3 superposition pulses for low and MB10 PINS pulses for large MB factors only and compare different initialization methods (SUP, SUP VERSE, PINS, PINS VERSE and MultiPINS) for the intermediate MB5 case. The root flipping pulse is excluded from this comparison and investigated separately for MB6 as it creates refocusing profiles with a non linear phase.

The results show that the proposed optimal control model and method can reduce the pulse power dramatically. The overall power of the initial pulses was reduced by roughly 58 percent for SUP MB3 and 52 percent for PINS MB10 (and by 77 percent for the RF MB6 pulse with a higher tolerance $e_{\text {in }}$ and $e_{\text {out }}$, see Tab. 1). Please note that the initial power of the PINS pulse 

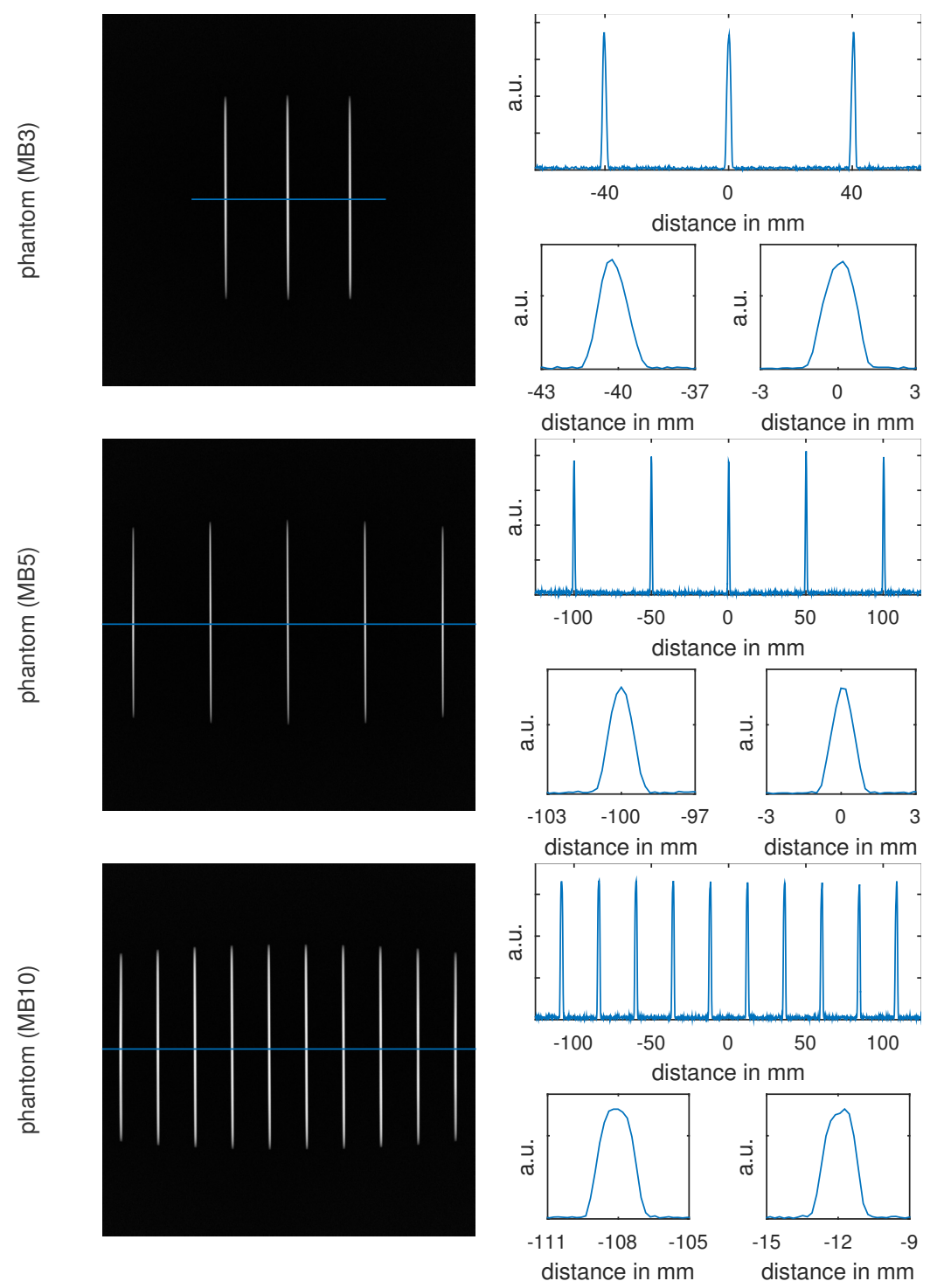

Figure 6: Reconstructed experimental data for the optimized MB3, MB5 and MB10 candidates.

already lies in the range of the MB3 pulse designed with superposition. As can be seen in the Figures 1-5 the optimal waveforms vary in their shape, and hence depend on the initialization. The figures show that the maximum and mean $B_{1}$ amplitude is reduced and the slice-selective gradient is modulated. While the initial PINS already uses a time varying Gs, the optimized superposition and in particular the optimized root flipped example introduce fluctuating Gs curves from constant initializations. The extent of the fluctuations in Gs changes with the prescribed maximum gradient slew rate, see Fig.3, and small fluctuations give a slightly reduced but still significant reduction in the pulse power, see Tab. 4. Even a constant and fixed Gs allows for a significant reduction of the pulse power for the root flipped initialization, but only a slight reduction for the superposition pulse, see Tab. 5. Keeping the non-constant Gs from PINS initialization fixed allows for nearly the same reduction of the pulse power as with the joint control. Accordingly, joint control is always beneficial, but the gain varys with the specific examples.

Five different initializations were compared for an intermediate $\mathrm{MB}$ factor of 5 with a uniform pulse duration and comparable error properties. The pulse power was heavily reduced in all cases while the maximal in-slice errors $\varepsilon_{\text {in }}$ remained the same. An increase in the out-of-slice errors $\varepsilon_{\text {out }}$ and phase errors $\varepsilon_{\text {ph }}$ was observed, but it remained below the prescribed error bounds of $2 \%$ and 0.01 radians, respectively. A superposition pulse with VERSE yielded $S \approx 0.56$ and 

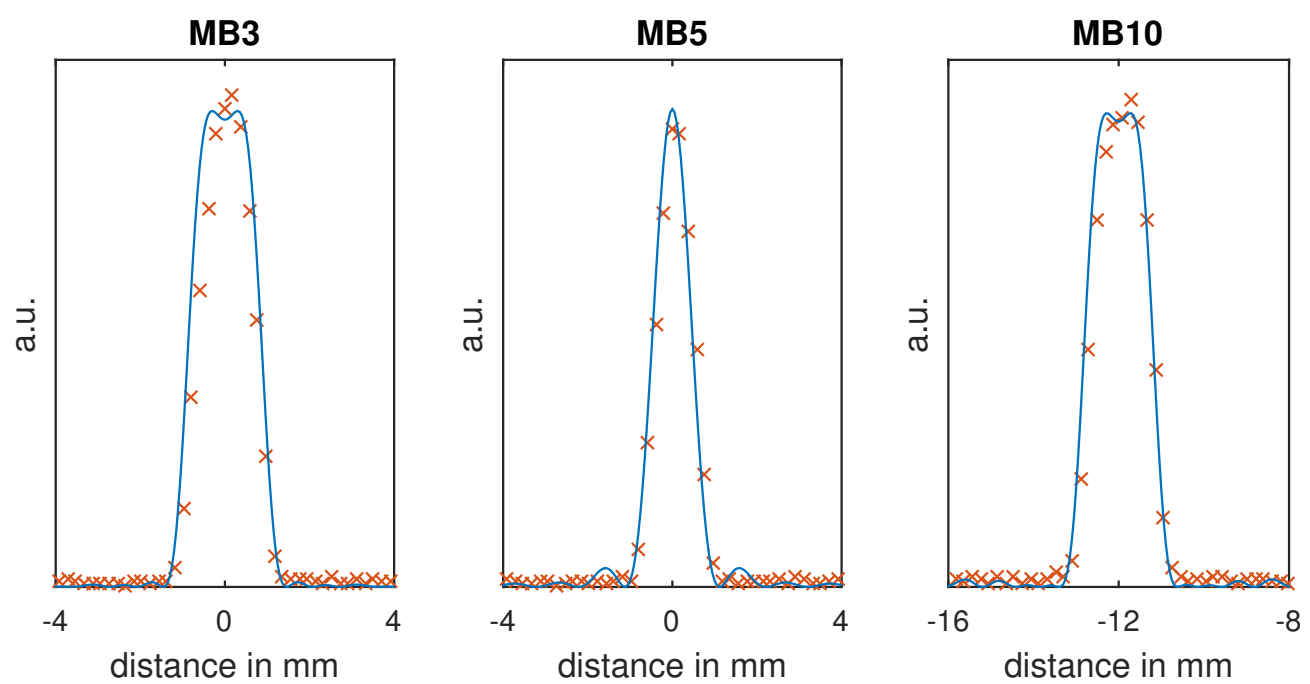

Figure 7: Simulated refocusing profile (solid line) and experimental phantom data (crosses) of the phantom measurements shown in Figure 6 for MB3 (zoom to one slice).
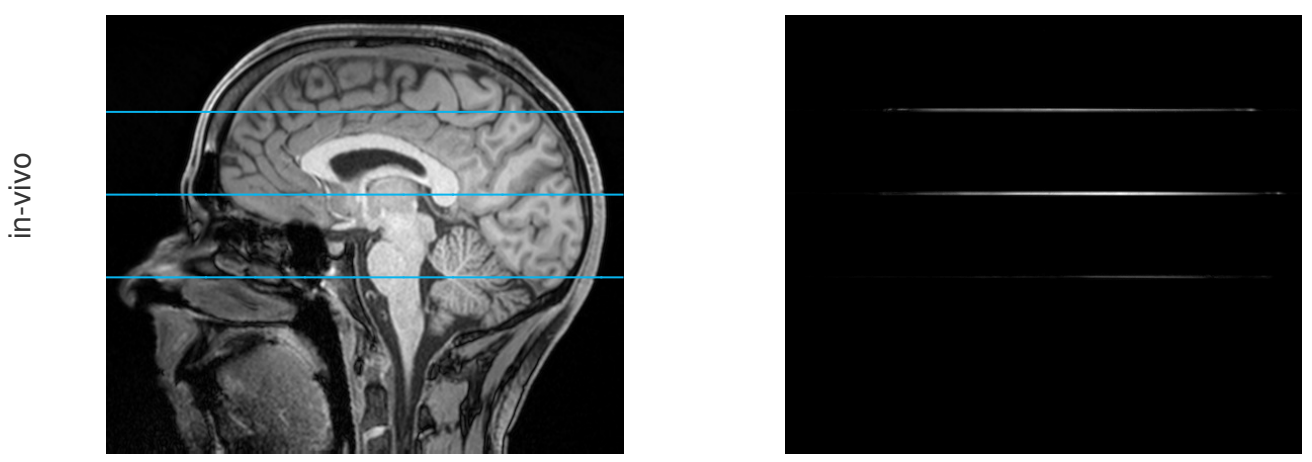

Figure 8: Reconstructed experimental data for in-vivo measurements using the optimized MB3 pulse.

therefore outperformed the same pulse without VERSE $(S \approx 0.77)$. However, much better power values of $S \approx 0.41$ were observed for optimized PINS, PINS with VERSE and MultiPINS pulses. Therefore, it seems that the optimal control method performs best with PINS based initializations, especially for large MB factors.

These improvements in the pulse power were possible by allowing a controlled deviation of $3 \%$ and $5 \%$ for the slice profile accuracy along with $2 \%$ and $4 \%$ for the out-of-slice region compared to an ideal rectangular refocusing with a transition zone excluded from the optimization. For two of the examples (SUP MB3 and PINS MB10) this formulation results in a slightly steeper refocusing profile for the optimized refocusing compared to the initial. Figures 1-5 show that the controlled deviation is not exceeded anywhere in the observation domain. In other words, the profile constraints are fulfilled exactly, which is an achievement of the presented penalization technique.

Two second-order methods of numerical optimal control were introduced, that both use adjointbased exact discrete derivatives, also for the second derivative. The precise derivative information allows for better progress when approaching the minimizer. For robustness of the optimization method w.r.t. the initialization and the problem parameters we embedded the Newton-type method into a trust-region framework using the Steihaug-CG method. Additionally, SteihaugCG reduces the computational effort of Newton-type methods, since it saves many Hessian evaluations in the first phase of the optimization. In the second phase of the optimization both methods profit from an increased convergence order compared to first-order methods. To save 
computational effort, both methods were applied as a Newton-Krylov method with a matrix-free evaluation of the Hessian. By semismooth Newton techniques the inequality constraints on the control variables were included into the optimization without increasing the numerical effort. The parameters of the optimization method were adapted automatically, or calibrated in the numerical experiments shown above. With these parameters the semismooth quasi-Newton method yields good results in short computational time. In contrast, the semismooth Newton method which uses increased computation time gives the best results.

Two examples for turbo spin echo sequences included explicit phase constraints, whereas the two other examples, intended for double refocused diffusion sequences, did not constrain the phase of the refocusing profile. The influence of the phase constraints on the optimal solutions is less severe than expected and the optimized cases lead to similar power reductions in the range of $50 \%$ for the standard constraints (Tab. 4).

The prescribed amplitude constraints on the optimized RF pulse, the gradient and its slew rate ensure practical applicability on MR scanners. Due to the semismooth Newton method as well as the penalization technique, all these constraints are never exceeded in the optimum. More closely, the results show that the maximum values for the $B_{1}$ amplitude and gradient strength were not attained in the optimal solution. This is due to the fact, that the reduction of the pulse power and the boundary conditions for the gradient already pull the maximum value downwards. However, both these constraints become active for examples with smaller maximum values, different boundary conditions, and especially for smaller pulse durations. In contrast, we observe that the slew rate constraint is active in any of the investigated scenarios, which can e.g. be seen in Fig. 3. A nonnegative minimum gradient value is also in effect for large pulse durations, as can be seen in Fig. 4 . If the minimum gradient value gets too large, the possible reduction of the pulse power is decreased significantly, as can be seen for $g_{\min }=2.31$ in Tab. 4 .

The pulses were designed based on the spin-domain description neglecting the relaxation effects. To justify this simplification we performed full Bloch simulations [14] for the optimized pulses and compared the simulations to the spin-domain results. The relaxation times were set to those of the cylindric phantom $\left(T_{1}=102 \mathrm{~ms}, T_{2}=81 \mathrm{~ms}\right)$ used in the experiment. In all simulations, the refocusing profiles of the optimized examples do not degenerate significantly in the presence of relaxation. We see a simple scaling of the refocusing profiles, similar to conventional RF pulses for short $T_{2}$ values. The effects of $T_{1}$ is an increase of the refocusing error outside of the slices, which is compensated by the $T_{2}$ relaxation and remains below the allowed deviation for each example. Since the $T_{1}$ relaxation times of typical in-vivo tissue are even higher and their influence on the refocusing profiles are negligible. These findings have been verified by the experimental phantom measurements.

The experimental validation was done on a $3 \mathrm{~T}$ scanner exchanging the excitation and refocusing pulse of a standard crushed spin echo sequence for a cylindrical phantom (MB3, $\mathrm{MB}_{5}$ and $\left.\mathrm{MB} 1 \mathrm{O}\right)$ and in-vivo $\left(\mathrm{MB}_{3}\right)$. Changing the readout gradient to the slice direction allows for a direct measurement of the simultaneous excited and refocused slices. The small inter slice ripples of the optimized refocusing pulses can be neglected for a real measurement as standard excitation pulses produce no marked transversal magnetization at these inter slice positions and the refocused magnetization would be weighted by a factor of $\sin (\theta / 2)^{2}$ with a spatially dependent but low inter slice refocusing flip angle $\theta$. Due to the adequate choice and implementation of the slew rate constraint, the fluctuating Gs shapes were implementable, and the measured slice profiles fit very well to the Bloch simulations for all measurements. The measured whole body SAR includes the non optimized excitation pulse and is therefore lower than the calculated power reduction of the optimized MB3 refocusing pulse. The good accordance of the simulations and the measurements shows the practical applicability of the optimized pulses on a standard clinical scanner. Using the specific hardware constraints of the desired MR system simplifies 
the application of optimized pulses since no further modifications or transformations have to be done, that moreover could reduce the optimality.

In simulations we compared the robustness of the optimized pulses w.r.t. inhomogeneities in $B_{0}$ and $B_{1}$ to the one of the initial pulses. The initial pulse and gradient pairs have a constant k-space excitation velocity and constant $B_{0}$ inhomogeneities lead to a constant spatial shift. Despite the time varying slice-selective gradient of the optimized examples, which is known to be prone to off-resonance effects, the profile fidelity remains almost unchanged in the range of $\pm 500 \mathrm{~Hz}$ and the impact of the chemical shift $(400 \mathrm{~Hz}$ at $3 \mathrm{~T})$ is negligible. For applications with larger $B_{0}$ inhomogeneities, the minimal amplitude constraint of the slice selective gradient can be increased or Gs can be fixed to enhance off-resonance robustness at the cost of a decreased power reduction. To investigate the influences of a temporal mismatch between the RF and slice selective gradient and eddy currents on the refocusing profiles, Bloch simulations were performed using a temporal shift of the slice selective gradient shape with respect to the RF pulse (up to $10 \mu \mathrm{s}$ ) and a first order low pass filtering (normalized numerator and denominator of 0.5 ) of the slice selective gradient shape. Both effects led to a symmetric decrease of the refocusing efficiency in dependence of the distance to the isocenter. Compared to the used initials with constant or repetitive gradient functions, the fast changes in the optimized slice selective gradient are more prone to these effects. However, such a behavior is not observable in the experimental phantom measurements and leads to the conclusion that the optimized examples can be successfully implemented on the used MR scanner without special compensation techniques. To decrease the sensitivity of gradient imperfections of the optimized results, it should be possible to incorporate this directly in the optimization by enforcing a smoothness constraint on the slice selective gradient or to directly include the gradient impulse response [35]. In the context of $B_{1}$ inhomogeneities all optimized examples behave similar as the initial pulses and lead to a smooth transition of higher and lower flip angles for a static $B_{1}$ increase or reduction, respectively. Furthermore, we do not expect an additional proneness to small in-plane and through-plane motion compared to conventional RF pulses. The same holds for movements between the excitation and the read-out. However, to reduce the influences of motion artifacts it should be considered to reduce the overall refocusing time.

If the pulse power is not the primary restriction, then the pulse duration can be decreased instead while keeping the pulse power. The reduction of the refocusing time is of high importance in SMS imaging and was chosen to be the topic of the 2015 ISMRM Challenge [13]. In this direction the next step is to minimize the pulse duration by time-optimal control [27] in order to find the shortest possible pulses that fulfill the given inequality constraints.

\section{Conclusions}

The modeling of the profile accuracy with inequality constraints together with a customized solution method allows for the computation of optimal RF pulses and slice-selective gradients, that outperform other approaches in the performance index. Moreover, the usage of constrained optimization guarantees for practical applicability and a direct implementation in clinical MR sequences.

\section{Acknowledgments}

This work is partially funded by the Austrian Science Fund (FWF) in the context of project "SFB F3209-18" (Mathematical Optimization and Applications in Biomedical Sciences). Partial support from BioTechMed-Graz and NAWI Graz is gratefully acknowledged. The authors thank 
Christian Clason from the University of Duisburg-Essen for many fruitful discussions on the topic of the paper.

\section{A. Lagrange calculus in the spin domain}

The adjoint based derivative is set up using a real-valued Lagrangian together with the Wirtinger calculus. Therefore we introduce the complex RF pulse $U_{m}=u_{m}+i v_{m}=r_{m} e^{i \vartheta_{m}}$. The Lagrange multipliers for the discrete Bloch equation (1) are called $p_{m}, q_{m} \in \mathbb{C}$ for $m=1, \ldots, N_{t}$. The control constraints (5) are kept explicitely. The Lagrangian of the penalized problem is defined in the usual way depending on the control variables $r_{m}, \vartheta_{m}, g_{m}$, the state variables $a_{m}, b_{m}$ and the Lagrange multipliers $p_{m}, q_{m}$ as

$$
\begin{aligned}
\mathcal{L} & =\frac{\tau}{2} \sum_{m=1}^{N_{t}} r_{m}^{2}+\zeta \vartheta_{m}^{2}+\zeta g_{m}^{2}+\frac{\delta \mu_{\text {out }}}{2 p} \sum_{z_{j} \in \Omega_{\text {out }}}\left(\frac{\left|b_{N_{t}}\right|^{2}}{e}\right)^{p}+\frac{\delta \mu_{\text {in }}}{2 p} \sum_{z_{j} \in \Omega_{\text {in }}}\left(\frac{\left|b_{N_{t}}\right|^{2}-1}{e}\right)^{p} \\
& +\frac{\delta \mu_{p}}{p} \sum_{l=1}^{L} \sum_{z_{j} \in S_{l}}\left(\frac{\varphi-\bar{\varphi}_{l}}{e_{p}}\right)^{p}+\frac{\tau \mu_{g}}{p} \sum_{m=2}^{N_{t}}\left(\frac{g_{m}-g_{m-1}}{\tau s_{\max }}\right)^{p} \\
& -\sum_{z_{j} \in \Omega} \mathfrak{R}\left\{\sum_{m=1}^{N_{t}}\left(a_{m}-\alpha_{m} a_{m-1}+\beta_{m}^{*} b_{m-1}\right) p_{m}^{*}+\left(b_{m}-\beta_{m} a_{m-1}-\alpha_{m}^{*} b_{m-1}\right) q_{m}^{*}\right\} .
\end{aligned}
$$

A compact form of the first-order necessary conditions can be derived efficiently using the Wirtinger calculus. In particular, for a Lagrangian of the form $\mathcal{L}=\mathfrak{R}\left(\mathcal{L}_{\mathbb{C}}\right)$ with a complexvalued expression $\mathcal{L}_{\mathbb{C}}$ the derivatives w.r.t the real and imaginary part $Z_{1}, Z_{2}$ of a complex variable $Z=Z_{1}+i Z_{2}$ can be combined to

$$
\frac{\partial \mathcal{L}}{\partial Z_{1}}+i \frac{\partial \mathcal{L}}{\partial Z_{2}}=\frac{\partial \mathcal{L}_{\mathbb{C}}}{\partial Z^{*}}+\left(\frac{\partial \mathcal{L}_{\mathbb{C}}}{\partial Z}\right)^{*}
$$

using the Wirtinger derivatives [11] of a function $f: \mathbb{C} \rightarrow \mathbb{C}, Z=Z_{1}+i Z_{2} \mapsto f(Z)$

$$
\frac{\partial f}{\partial Z}=\frac{1}{2}\left(\frac{\partial f}{\partial Z_{1}}-i \frac{\partial f}{\partial Z_{2}}\right), \quad \frac{\partial f}{\partial Z^{*}}=\frac{1}{2}\left(\frac{\partial f}{\partial Z_{1}}+i \frac{\partial f}{\partial Z_{2}}\right) .
$$

In our case $\mathcal{L}_{\mathbb{C}}$ does not depend on $p_{m}, q_{m}, a_{m}^{*}, b_{m}^{*}$. Therefore the state equations (1) are easily recovered by differentiation w.r.t. $p_{m}^{*}, q_{m}^{*}$. Analogously differentiating w.r.t. $a_{m}$ and $b_{m}$ and complex conjugation gives the adjoint Bloch equations

$$
\begin{aligned}
& p_{m}=\alpha_{m+1}^{*} p_{m+1}+\beta_{m+1}^{*} q_{m+1}, \\
& q_{m}=-\beta_{m+1} p_{m+1}+\alpha_{m+1} q_{m+1},
\end{aligned}
$$

for $m=1, \ldots, N_{t}-1$ and pointwise in each $z_{j} \in \Omega$. For the terminal condition in case of a refocusing pulse both Wirtinger derivatives w.r.t. $b_{N_{t}}$ and $b_{N_{t}}^{*}$ have to be evaluated. Depending on the location $z_{j}$ we find $p_{N_{t}}=0$ and

$$
\begin{aligned}
q_{N_{t}} & =\frac{\mu_{\text {out }} \delta}{e} \chi_{\Omega_{\text {out }}}\left(\frac{\left|b_{N_{t}}\right|^{2}}{e}\right)^{p-1} b_{N_{t}} \\
& +\frac{\mu_{\text {in } \delta}}{e} \chi_{\Omega_{\text {in }}}\left(\frac{\left|b_{N_{t}}\right|^{2}-1}{e}\right)^{p-1} b_{N_{t}}+\left\{\sum_{l=1}^{N_{\mathrm{MB}}} \chi_{S_{l}} \frac{\delta \mu_{p}}{e}\right. \\
& {\left.\left[\left(\frac{\varphi-\bar{\varphi}_{l}}{e_{p}}\right)^{p-1}-\frac{1}{N_{l}} \sum_{z_{k} \in S_{l}}\left(\frac{\varphi\left(z_{k}\right)-\bar{\varphi}_{l}}{e_{p}\left(z_{k}\right)}\right)^{p-1}\right]\right\} 2 \frac{i b_{N_{t}}}{b_{N_{t}}^{*} b_{N_{t}}} . }
\end{aligned}
$$


The last fraction stems from the chain rule using the derivative of the arctan function in $\varphi=\arg \left(b_{N_{t}}^{2}\right)$. Moreover, $\chi_{S_{l}}$ is the characteristic function of the in-slice set $S_{l}=\left\{z_{1}^{l}, \ldots, z_{N_{l}}^{l}\right\}$ with $N_{l}$ points and mean phase $\bar{\varphi}_{l}=\sum_{k=1}^{N_{l}} \varphi\left(z_{k}^{l}\right) / N_{l}$.

The gradient of the reduced objective $\mathbf{j}^{\prime}=E \mathbf{x}+\mathbf{F}^{\prime}(\mathbf{x})$ is given by the partial derivatives w.r.t the control variables. Its components are given by chain rule for the polar coordinates

$$
\begin{aligned}
& \frac{\partial \mathcal{L}}{\partial r_{m}}=\tau r_{m}+\frac{\partial \mathcal{L}}{\partial u_{m}} \cos \left(\vartheta_{m}\right)+\frac{\partial \mathcal{L}}{\partial v_{m}} \sin \left(\vartheta_{m}\right), \\
& \frac{\partial \mathcal{L}}{\partial \vartheta_{m}}=\zeta \tau \vartheta_{m}-\frac{\partial \mathcal{L}}{\partial u_{m}} r_{m} \sin \left(\vartheta_{m}\right)+\frac{\partial \mathcal{L}}{\partial v_{m}} r_{m} \cos \left(\vartheta_{m}\right),
\end{aligned}
$$

for $m=1, \ldots, N_{t}$ and by $\frac{\partial \mathcal{L}}{\partial g_{m}}, m=2, \ldots, N_{t}-1$, which is computed below. Each first term is collected in $E \mathbf{x}$, and the other terms constitute $\mathbf{F}^{\prime}(\mathbf{x})$. In these equations we need

$$
\frac{\partial \mathcal{L}}{\partial u_{m}}=\mathfrak{R}\left(\frac{\partial \mathcal{L}_{\mathbb{C}}}{\partial U_{m}}+\frac{\partial \mathcal{L}_{\mathbb{C}}}{\partial U_{m}^{*}}\right), \quad \frac{\partial \mathcal{L}}{\partial v_{m}}=\mathfrak{J}\left(\frac{\partial \mathcal{L}_{\mathbb{C}}}{\partial U_{m}^{*}}-\frac{\partial \mathcal{L}_{\mathbb{C}}}{\partial U_{m}}\right)
$$

With $\frac{\partial \alpha_{m}^{*}}{\partial \phi_{m}}=\left(\frac{\partial \alpha_{m}}{\partial \phi_{m}}\right)^{*}$ and analogously for $\beta_{m}$ the result is

$$
\begin{array}{rlrl}
\frac{\partial \mathcal{L}}{\partial u_{m}} & =\gamma^{2} \tau^{2} u_{m} R_{m}-\gamma \tau \mathfrak{I}\left(Q_{m}\right), & m & =1, \ldots, N_{t}, \\
\frac{\partial \mathcal{L}}{\partial v_{m}}=\gamma^{2} \tau^{2} v_{m} R_{m}-\gamma \tau \mathfrak{R}\left(Q_{m}\right), & m=1, \ldots, N_{t},
\end{array}
$$

with

$$
\begin{aligned}
Q_{m} & =\sum_{z_{j} \in \Omega} \sin \left(\phi_{m} / 2\right) \frac{1}{\phi_{m}}\left(a_{m-1} q_{m}^{*}-b_{m-1}^{*} p_{m}\right), \\
R_{m} & =\sum_{z_{j} \in \Omega} \frac{1}{\phi_{m}} \mathfrak{R}\left\{\left(a_{m-1} p_{m}^{*}+b_{m-1}^{*} q_{m}\right) \frac{\partial \alpha_{m}}{\partial \phi_{m}}\right. \\
& \left.+\left(a_{m-1} q_{m}^{*}-b_{m-1}^{*} p_{m}\right) \frac{\partial \beta_{m}}{\partial \phi_{m}}\right\}, \\
\frac{\partial \alpha_{m}}{\partial \phi_{m}}= & i \frac{\gamma \tau z g_{m}}{\phi_{m}}\left(\frac{\cos \left(\phi_{m} / 2\right)}{2}-\frac{\sin \left(\phi_{m} / 2\right)}{\phi_{m}}\right)-\frac{\sin \left(\phi_{m} / 2\right)}{2}, \\
\frac{\partial \beta_{m}}{\partial \phi_{m}}= & i \frac{\gamma \tau\left(u_{m}+i v_{m}\right)}{\phi_{m}}\left(\frac{\cos \left(\phi_{m} / 2\right)}{2}-\frac{\sin \left(\phi_{m} / 2\right)}{\phi_{m}}\right) .
\end{aligned}
$$

Differentiation w.r.t. $g_{m}$ gives finally

$$
\begin{aligned}
\frac{\partial \mathcal{L}}{\partial g_{m}} & =\zeta \tau g_{m}+\sum_{z_{j} \in \Omega} \mathfrak{R}\left\{\left(a_{m-1} p_{m}^{*}+b_{m-1}^{*} q_{m}\right) \frac{\partial \alpha_{m}}{\partial g_{m}}\right. \\
& \left.+\left(a_{m-1} q_{m}^{*}-b_{m-1}^{*} p_{m}\right) \frac{\partial \beta_{m}}{\partial g_{m}}\right\} \\
& +\frac{\mu_{g}}{s_{\max }}\left(\frac{g_{m}-g_{m-1}}{\tau s_{\max }}\right)^{p-1}-\frac{\mu_{g}}{s_{\max }}\left(\frac{g_{m+1}-g_{m}}{\tau s_{\max }}\right)^{p-1}
\end{aligned}
$$

for $m=2, \ldots, N_{t}-1$ and with

$$
\begin{aligned}
& \frac{\partial \alpha_{m}}{\partial g_{m}}=\frac{\gamma \tau z}{\phi_{m}}\left(i \sin \left(\phi_{m} / 2\right)+\gamma \tau z g_{m} \frac{\partial \alpha_{m}}{\partial \phi_{m}}\right), \\
& \frac{\partial \beta_{m}}{\partial g_{m}}=\frac{(\gamma \tau z)^{2}}{\phi_{m}} g_{m} \frac{\partial \beta_{m}}{\partial \phi_{m}} .
\end{aligned}
$$




\section{References}

[1] C. S. Aigner, C. Clason, A. Rund, and R. Stollberger. Efficient high-resolution RF pulse design applied to simultaneous multi-slice excitation. fournal of Magnetic Resonance, 263:33 - 44, 2016. doi:http://dx.doi.org/10.1016/j. jmr.2015.11.013.

[2] E. J. Auerbach, J. Xu, E. Yacoub, S. Moeller, and K. Uğurbil. Multiband accelerated spin-echo echo planar imaging with reduced peak RF power using time-shifted RF pulses. Magnetic Resonance in Medicine, 69(5):1261-1267, 2013. doi:10.1002/mrm. 24719.

[3] M. Barth, F. Breuer, P. Koopmans, D. Norris, and B. Poser. Simultaneous multislice (sms) imaging techniques. Magnetic Resonance in Medicine, 81:63-81, 2016. doi: 10.1002/mrm. 25897.

[4] M. Bergounioux, M. Haddou, M. Hintermüller, and K. Kunisch. A comparison of a MoreauYosida based active set strategy and interior point methods for constrained optimal control problems. SIAM fournal on Optimization, 11:495-521, 2000. 10.1137/S1052623498343131. doi: $10.1137 /$ S1052623498343131.

[5] M. Bergounioux, K. Ito, and K. Kunisch. Primal-dual strategy for constrained optimal control problems. SIAM Journal on Control and Optimization, 37:1176-1194, 1999. 10.1137/So363012997328609. doi: 10.1137/S0363012997328609.

[6] M. Bernstein, K. King, and X. Zhou. Handbook of MRI Pulse Sequences. Elsevier Academic Press, 2004. doi: http://dx. doi.org/10.1016/B978-012092861-3/50003- 0.

[7] R. H. Byrd, J. Nocedal, and R. B. Schnabel. Representations of quasi-newton matrices and their use in limited memory methods. Mathematical Programming, 63(1):129-156, 1994. URL: http://dx.doi.org/10.1007/BF01582063, doi:10.1007/BF01582063.

[8] S. Conolly, D. Nishimura, and A. Macovski. Variable rate selective excitation. IEEE Trans. "Medical Imaging", MI-5:106, 1986.

[9] S. Conolly, D. Nishimura, A. Macovski, and G. Glover. Variable-rate selective excitation. Journal of Magnetic Resonance, 78(3):440-458, 1988.

[10] C. Eichner, L. L. Wald, and K. Setsompop. A low power radiofrequency pulse for simultaneous multislice excitation and refocusing. Magnetic Resonance in Medicine, 72(4):949-958, 2014. doi : 10.1002/mrm. 25389.

[11] W. Fischer and I. Lieb. A Course in Complex Analysis. Vieweg+Teubner Verlag, 2012. doi : 10.1007/978-3-8348-8661-3.

[12] W. A. Grissom, G. C. McKinnon, and M. W. Vogel. Nonuniform and multidimensional Shinnar-Le Roux RF pulse design method. Magnetic Resonance in Medicine, 68(3):690-702, 2012. doi:10.1002/mrm. 23269.

[13] W. A. Grissom, K. Setsompop, S. A. Hurley, J. Tsao, J. V. Velikina, and A. A. Samsonov. Advancing RF pulse design using an open-competition format: Report from the 2015 ISMRM challenge. Magnetic Resonance in Medicine, pages n/a-n/a. URL: http://dx.doi.org/10. 1002/mrm. 26512, doi : 10.1002/mrm. 26512.

[14] B. Hargreaves. Bloch equation simulation. 2003. URL: http://mrsrl.stanford.edu/ -brian/blochsim/.

[15] B. A. Hargreaves, C. H. Cunningham, D. G. Nishimura, and S. M. Conolly. Variable-rate selective excitation for rapid mri sequences. Magnetic Resonance in Medicine, 52(3):59o-597, 2004. URL: http://dx.doi .org/10.1002/mrm. 20168, doi : 10.1002/mrm. 20168. 
[16] M. Hintermüller, K. Ito, and K. Kunisch. The primal-dual active set method as a semi-smooth Newton method. SIAM fournal on Optimization, 13(3):865-888, 2003. 10.1137/S1052623401383558. doi:10.1137/S1052623401383558.

[17] M. Hintermüller and K. Kunisch. Stationary optimal control problems with pointwise state constraints. In Numerical PDE Constrained Optimization, volume 72 of Lecture Notes in Computational Science and Engineering. Springer, Berlin, 2009.

[18] K. Ito and K. Kunisch. Lagrange Multiplier Approach to Variational Problems and Applications. SIAM, 2008.

[19] E. Jaynes. Matrix treatment of nuclear induction. Physical Review, 98(4):1099-1105, 1955.

[20] N. Kobayashi, K. Ugurbil, and X. Wu. Shortening nonlinear phase multiband refocusing pulses with verse. In Proc. Intl. Soc. Mag. Reson. Med. 24, page 3253, 2016.

[21] M. Lapert, Y. Zhang, M. A. Janich, S. J. Glaser, and D. Sugny. Exploring the physical limits of saturation contrast in magnetic resonance imaging. Scientific Reports, 2, 2012. doi: $10.1038 /$ s rep00589.

[22] S. Mueller. Multifrequency selective RF pulses for multislice MR imaging. Magnetic Resonance in Medicine, 6(3):364-371, 1988. doi:10.1002/mrm. 1910060315.

[23] J. Nocedal and S. J. Wright. Numerical Optimization. Springer Series in Operations Research and Financial Engineering. Springer, New York, 2nd edition, 2006. 10.1007/978-0-387-400655. doi : 10. 1007/978- - - - 387-40065- 5 .

[24] D. G. Norris, P. J. Koopmans, R. Boyacioğlu, and M. Barth. Power independent of number of slices (PINS) radiofrequency pulses for low-power simultaneous multislice excitation. Magnetic Resonance in Medicine, 66(5):1234-1240, 2011. doi:10.1002/mrm. 23152.

[25] J. Pauly, P. Le Roux, D. Nishimura, and A. Macovski. Parameter relations for the Shinnar-Le Roux selective excitation pulse design algorithm. Medical Imaging, IEEE Transactions on, 10(1):53-65, 1991. doi: 10.1109/42.75611.

[26] K. Pieper. Finite element discretization and efficient numerical solution of elliptic and parabolic sparse control problems. PhD Dissertation, Technische Universität München, April 2015.

[27] A. Rund, C. Aigner, K. Kunisch, and R. Stollberger. Simultaneous multislice refocusing by time-optimal control. In Proc. ISMRM 25, 2017.

[28] A. Sharma, M. Lustig, and W. A. Grissom. Root-flipped multiband refocusing pulses. Magnetic Resonance in Medicine, 75(1):227-237, 2016. doi : 10.1002/mrm . 25629.

[29] T. Steihaug. The conjugate gradient method and trust regions in large scale optimization. SIAM Journal on Numerical Analysis, 20:626-637, 1983.

[30] M. Ulbrich. Semismooth Newton methods for operator equations in function spaces. SIAM fournal on Optimization, 13(3):805-841, 2003. doi : 10.1137/S1052623400371569.

[31] M. Ulbrich. Semismooth Newton Methods for Variational Inequalities and Constrained Optimization Problems in Function Spaces. MOS-SIAM Series on Optimization. SIAM, 2011.

[32] M. Ulbrich, S. Ulbrich, and M. Heinkenschloss. Global convergence of trust-region interiorpoint algorithms for infinite-dimensional nonconvex minimization subject to pointwise bounds. SIAM fournal on Control and Optimization, 37(3):731-764, 1999. doi:10.1137/ S0363012997325915.

[33] M. S. Vinding, I. I. Maximov, Z. Tošner, and N. C. Nielsen. Fast numerical design of spatialselective RF pulses in MRI using Krotov and quasi-Newton based optimal control methods. fournal of Chemical Physics, 137(5):054203, 2012. doi : 10.1063/1.4739755. 
[34] E. Wong. Optimized phase schedules for minimizing peak RF power in simultaneous multi-slice RF excitation pulses. In Proc. ISMRM 2O, page 2209, 2012.

[35] M. Çavuşoğlu, R. Mooiweer, K. P. Pruessmann, and S. J. Malik. Verse-guided parallel rf excitations using dynamic field correction. NMR in Biomedicine, 30(6):e3697-n/a, 2017. e3697 NBM-16-oo8o.R1. URL: http://dx.doi.org/10.1002/nbm.3697, doi:10.1002/ nbm. 3697. 